\title{
EL VOCABULARIO DE LAS ARMAS EN MICÉNICO
}

\author{
POR
}

Alberto BernabÉ*

\section{RESUMEN - ABSTRACT}

Estudio sistemático de la creación, estructura y desarrollo del vocabulario referido a las armas en las tablillas micénicas y en el griego posterior. Los resultados obtenidos por métodos lingüísticos o filológicos pueden así ser cotejados con los logrados por la arqueología.

Systematic analysis of rise, structure, and development of the words referred to weapons in Mycenaean tablets and in first Millennium Greek. The results of application of linguistic or philological methods can so be collated with ones achieved by archeologists.

\section{Palabras ClaVe - KeYwords}

Armas, historia militar, artesanía antigua, micénico, griego antiguo.

Weapons, military history, ancient craftmanship, Mycenaean, Ancient Greek

\section{INTRODUCCIÓN}

Pretendo en este trabajo examinar la creación, estructura y desarrollo del vocabulario de las armas documentado directa o indirectamente en micénico; en consecuencia, los resultados se derivarán de un análisis del léxico desde puntos de vista casi exclusivamente lingüísticos y filológicos ${ }^{1}$ : inventario de los términos, contextos en que aparecen, sistema que configuran, su origen y formación y su pervivencia o no en el primer milenio. En una fase posterior, los resultados obtenidos deberían contrastarse con los datos suministrados por la arqueología y la iconografía, para ver si el esquema propuesto tiene su correlato en los realia, pero esa constatación, naturalmente, corresponde a expertos en esos terrenos

* Departamento de Filología Griega y Lingüística Indoeuropea. Desp. 33. Facultad de Filología, UCM, 28040 Madrid.

1 Se trata de una metodología expuesta por F. Aura Jorro, "Cambios léxicos del micénico al griego del primer milenio", en E. De Miro, L. Godart, A. Sacconi (edd.), Atti e Memorie del Secondo Congresso Internazionale di Micenología (Roma-Napoli ottobre 1991), Roma 1996, pp. 177-188 y aplicada ya en trabajos anteriores, como A. Bernabé, "Estructura del léxico micénico sobre el carro y sus partes", en De Miro, Godart, Sacconi (edd.), Atti e Memorie... cit., pp. 195-207; A. Bernabé et alii, "Estudios sobre el vocabulario micénico I: términos referidos a las ruedas", Minos 25-26, 1990-1991, pp. 133-173; "Estudios sobre el vocabulario micénico II: Términos referidos a los carros", Minos 27-28, 1992-1993, pp. 125-166. 
El vocabulario de las armas ha sido reiteradamente estudiado desde diversos puntos de vista y la mayoria de sus términos se ha determinado con gran precisión y seguridad, por lo que caben escasos puntos de discusión ${ }^{3}$. No obstante, pienso que el análisis desde un punto de vista lexicográfico, su examen como un conjunto, puede aportar algunos detalles nuevos al cuadro adquirido.

\section{DESIGNACIONES GENÉRICAS}

\subsection{El equipo}

El 'equipo' o 'armamento' de un soldado recibía probablemente el nombre de $\theta \in \hat{\cup} \chi 0 S^{4}$ (griego alfabético $t \in \hat{X} X 0 S$ ). Se documenta en micénico por vía indirecta en una tablilla de

2 Cf. fundamentalmente: H. Trümpy, Kriegerische Fachausdrücke im Griechischen Epos, Basel 1950; L. Palmer, The interpretation of Mycenaean Greek texts, Oxford 1963, pp. 314-337; A. M. Snodgrass, "The Linear B arms and armour tablets-again", Kadmos 4, 1965, pp. 96-110; M. Kostorou, "Mycenaean offensive weapons", AAA 5, 1972, pp. 325-337; M. Lejeune, "La civilisation mycénienne et la guerre", Mémoires de Philologie Mycénienne III, Roma 1972, pp. 57-77; P. Càssola Guida, Le armi difensive dei micenei nelle figurazioni, Roma 1973; M. Ventris y J. Chadwick, Documents in Mycenaean Greek, $2^{\mathrm{a}}$ ed. por J. Chadwick, Cambridge 1973, pp. 375-381 y 523-524 (a partir de ahora citado Docs.); F. Ferluga, "La terminologia delle armi nell'onomastica micenea", ZAnt 25, 1975, pp. 381-387; Y. Duhoux, Aspects du vocabulaire économique micénien, Amsterdam 1976, pp. 123-128; F. Matz - H.-G. Buchholz (eds.), Archaeologia Homerica. I. Kriegswesen. Teil 1. Schutzwaffen und Wehrbauten. Göttingen 1977; A. Franceschetti, "Armi e guerra in testi micenei", RAAN 53, 1978, pp. 67-90; F. Ferluga, I nomi delle armi in miceneo, Skopje 1979; F. Vandenabeele - J.-P. Olivier, Les idéogrammes archéologiques du Linéaire B, Paris, 1979, cap.

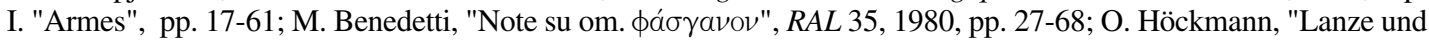
Speer im Spätminoischen und mykenischen Griechenland", Jahrbuch des Romisch-Germanischen Zentralmuseums Mainz 21, 1980, pp. 13-158; R. A. J. Avila, Bronzene Lanzen- und Pfeilspitzen der griechischen Spätbronzezeit, München 1983; F. Gschnitzer, "Vocabulaire et institutions: la continuité historique du deuxième au premier millénaire", en A. Heubeck - G. Neumann (ed.), Res Mycenaeae, Göttingen 1983, pp. 109-134; J. L. Melena, "Further thoughts on Mycenaean o-pa", ibid. pp. 258-286; J. Driessen - C. F. Macdonald, "Some Military Aspects of the Aegean in the Late Fifteenth and Early Fourteenth Centuries B.C.", ABSA 79, 1984, pp. 49-74, (esp. C. Macdonald, "Aegean swords and warrior graves: their implications for Knossian military organization", pp. 56-74); A. XenakiSakellariou, "Poignées ouvragées d'épées et de poignards mycéniens", en Hommage à Henri van Effenterre, Paris 1984, pp. 129-139; I. Kilian-Dirlmeier, "Noch Einmal zu den 'Kriegergräbern' von Knossos", JRGZM 32, 1985, pp. 196-214; J. T. Killen, "New readings in the Linear B tablets from Knossos". Appendix by J. MacGillivray, "The discovery of a new fragment of tablets Ga(2) 420", Kadmos 24, 1985, pp. 26-33; A. Heubeck, "Mykenisch qe-ro ", en A. Etter (ed.), O-o-pe-ro-si. Festchrift fur Ernst Risch zum 75. Geburtstag, Berlin 1986, pp. 285-296; id., "Vermutungen zu KN L 693", Kadmos 25, 1986, pp. 141-146; L. Godart, "Le rôle du palais dans l'organization militaire mycénienne", en E. Lévy (ed.), Le système palatial en Orient, en Grèce et à Rome. Actes du Colloque de Strasbourg 19-22 juin 1985, Strasbourg 1987, pp. 237-253; L. Vagneti, "Armi per i Micenei", Archeo 41, 1988, pp. 21-27; L. Godart, A. Franceschetti, Appunti di filologia micenea, Napoli 1990, pp. 146-159; M. S. Ruipérez - J. L. Melena, Los griegos micénicos, Madrid 1990, pp. 204-207; M. del Freo, "wi-so-wo-pa-na o wi-so-wo-pa-to? Festschrift Bartoněk, Brno 1991, pp. 33-51; I. Hajnal, "Der Mykenische Personenname a-e-ri-qo-ta", en J.-P. Olivier (ed.), Mykenaïka, Actes du IXe Colloque International sur les textes mycéniens et égéens... (Athènes 2-6 octobre 1990), Paris 1992, pp. 285-301; S. Hiller, "The 'corridor of the sword tablets' and the 'Arsenal'. The evidence of the linear B Texts", ibid. pp. 303-314; O. Panagl, "Mykenisch und die Sprache Homers: alte Probleme- Neue Resultate", ibid., pp. 499513; M. del Freo, "Mic. qe-ro 2 : alcune precisazioni", en De Miro, Godart, Sacconi (edd.), Atti ... cit., pp. 237-244; P. Negri Scafa, "Paralleli tra gli archivi orientali e gli archivi micenei", ibid., pp. 375-378; Th. G, Palaima, "'Contiguities' in the linear B tablets from Pylos", ibid. 379-396; C.W. Shelmerdine, "From Mycenae to Homer: the next generation", ibid., pp. 467-478; E. Scafa, "L'epica omerica e l'organizzazione militare micenea", SMEA 33, 1994, pp. 5567; J. T. Killen, "Mycenaean opa" y "New readings and interpretations in the Pylos tablets", en S. Deger-Jalkotzky, S. Hiller y O. Panagl (edd.), Floreant Studia Mycenaea, Wien 1999, pp. 325-341 y 343-353. El autor me permitió consultar ambos cuando aún estaban inéditos, lo que le agradezco profundamente.

3 Tal como reconstruye la palabra, antes de la actuación de la ley de Grassmann, C. J. Ruijgh, "Observations sur les neutres en s/h", en A. Heubeck y G. Neumann (eds.), Res Mycenaeae, Akten des VII. Internationalen Mykenologischen Colloquiums in Nürnberg vom 6.-10 April 1981, Göttingen 1983, pp. 391-407: 395. 
Cnoso (KN V(1) 150 + 7624) en que leemos las palabras to-ko-so-ta, a-te-u-ke seguidas del

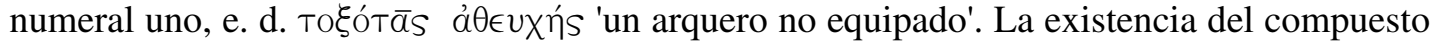

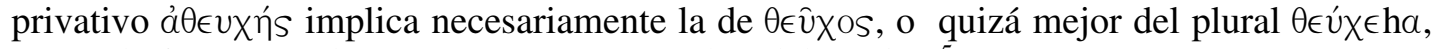
que es la forma usual en Homero, como nombre del 'equipo'5.

La palabra $\theta \in \hat{x} X 0 S$ deriva de una antigua raíz indoeuropea, de sentido muy general (P. $271^{6} *$ dheugh-), que da lugar a varios derivados, con diversos sentidos, entre ellos, dentro del propio griego, a los verbos $\tau \in u ́ x \omega$ 'preparar, producir, fabricar' (documentado en mic. en part.

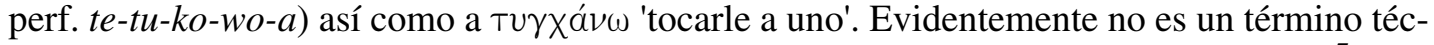
nico, sino una palabra de sentido amplio, que traduciríamos 'lo que toca o corresponde ${ }^{17}$, y es el que se emplea para referirse a un grupo variado de componentes que pertrechan una determinada realidad principal (persona o cosa) de forma que se considera completa y en condiciones para cumplir sus funciones. Ello explica que esta palabra, que hallamos reiteradamente utilizada en el primer milenio, admita múltiples traducciones, según la realidad a la que se aplica: utensilios, instrumentos, útiles, pertrechos, aparejos (de una nave), arreos (de una brida), etc.

\subsection{Las armas}

También podemos documentar indirectamente una palabra asimismo genérica para designar las 'armas', $€ \nu T t \in h a$. Formaría parte del nombre de oficio e-te-do-mo, que aparece cuatro veces en Pilo calificando a beneficiarios de un o-na-to en las tablillas del 'Catastro' (Ea 808, En 609, 5, Eo 211.2, Ep 301.5) y una vez, en plural en Cnoso (Uf 432.6) iniciando, tras una línea en blanco, una serie de antropónimos ${ }^{8}$.

Aunque se han hecho otras propuestas, menos verosímiles ${ }^{9}$, parece preferible entender $e$ -

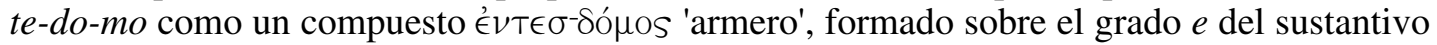

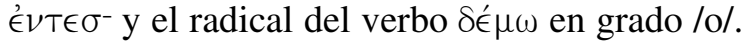

La etimología de $\epsilon^{\prime \prime} \nu T \in$ h $\alpha$ es oscura, pero parece ser indoeuropea, probablemente un derivado de la raíz ide. *sen- 'ganar, vencer, obtener como presa' (P. 906, cf. ai. sanóti 'ganar', gr.

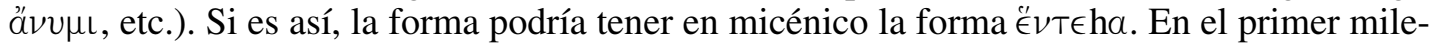
nio $\iota^{\prime \prime} \nu T \in \alpha$, usado en plural, conserva el sentido genérico de 'armas', alternando con el de 'arreos', 'arneses', 'utensilios'10.

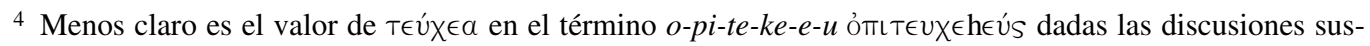
citadas en la interpretación concreta de este término, cf. F. Aura Jorro, Diccionario micénico Madrid I, 1985; II 1993 (a partir de ahora abreviado DMic.) s.v. y M. Guidi, "Miceneo i-qo, greco ïmmos", Minos 23, 1988, p. 169.

5 Aquí y en adelante, la abreviatura P. seguida de un número corresponde a J. Pokorny, Indogermanisches etymologisches Wörterbuch, Bern 1959 y el número de página.

${ }^{6}$ Y así lo hallamos en Pilo Ub 1315 en instrumental te-u-ke-pi para referirse a los elementos componentes de una brida.

7 Cf. bibliografía sobre las propuestas en DMic. s.v.

8 Cf. DMic. s.v.

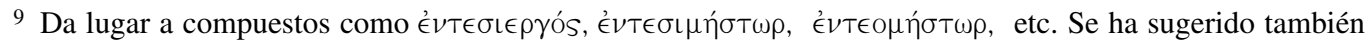
que esta misma palabra ${ }^{\prime \prime} \nu T \in$ ha podría formar parte del compuesto $e$-to-wo-ko(-i) nombre de oficio que aparece en singular una vez en Cnoso y otra en Pilo y dos veces en plural en Pilo. Según esta propuesta, deberíamos leer

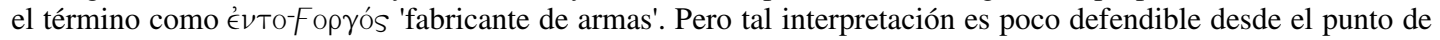
vista lingüístico, por lo que prefiero entenderlo como compuesto del adverbio évTós 'dentro', esto es, '́vTo-Fo $\rho^{-}$

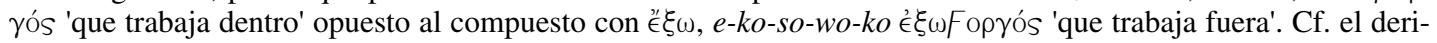
vado $€ \nu \nu T O \theta \in \nu$ donde hallamos la forma sin $-s$ - del adverbio. Cf. DMic ss. $v v$. para la bibliografía de las propuestas.
} 
Por último, podríamos documentar la palabra őt $\lambda \alpha$ en el antropónimo o-po-ro-me-no

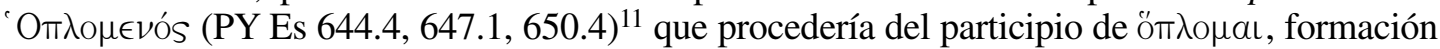
en grado /o/ correspondiente al verbo É $\pi \omega$ 'preparar, disponer'. Se trata de un término de procedencia indoeuropea, derivado de la raíz *sep- (P. 909, cf. ai. sápati, lat. sepelio, etc.).

\subsection{Las armas como despojo}

El micénico habría conocido el término para las armas tomadas al enemigo como despojo évapa, si el topónimo pilio e-na-po-ro (PY An 37.4, etc.) está formado efectivamente sobre esta palabra, igual que el epíteto de Ares évapoфópos (Hes. Scut. 192) ${ }^{12}$. Probablemente "̈v mencionada en $\S 2.2$ a propósito de $\epsilon^{\prime \prime} \nu T \epsilon \alpha$. Si es así, la palabra podría tener en mic. la forma *Évapa. Conservada en la épica, la palabra cae luego en desuso.

\section{ARMAS DEFENSIVAS}

\subsection{La serie Sc de Cnoso}

Con respecto a las armas defensivas, disponemos de documentación directa, ya que hay series de tablillas dedicadas a algunas de ellas.

Poca información lexicográfica nos brinda la serie cnosia Sc en que se enumeran equipos militares, concretamente carros, corazas y caballos, ya que cada tablilla sólo nos presenta un nombre propio seguido de los logogramas BIG, TUN y EQU (es decir, respectivamente, el carro con ruedas, la coraza y del caballo), acompañados de números.

\subsection{La serie pilia $\mathrm{Sh}$}

Más interesante es la serie pilia Sh, compuesta por doce tablillas escritas por la misma mano $^{13}$, halladas en la parte noroeste de la habitación aneja $7^{14}$. La situación es la siguiente:

a) Seis tablillas contienen la misma información. De ellas, una (PY Sh 737) presenta completas algunas palabras que en las demás aparecen sólo en abreviatura acrofónica, por lo que podría ser la que encabezara el grupo:

ARM 1 o-pa-wo-ta, me-zo-a,$O 20$ me-u-jo-a,$O 10$ ko-ru-to, $O 4$ pa-ra-wa-jo 2

Se trata de la referencia a una armadura, en la que se especifican los números de los elementos que la componen. Presento una transcripción convencional y una traducción, aunque para ello haya que adelantar algo de lo que luego se analizará con mayor detenimiento:

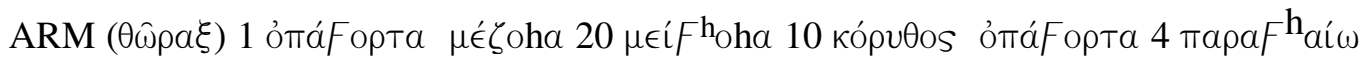
2

1 Armadura: apliques grandes 20; apliques pequeños 10; apliques del casco 4; orejeras 2.

Otras cinco (Sh 733, 741, 742, 743 y 744) presentan abreviaturas acrofónicas $O$ para $O$ pa-wo-ta (como en la primera), KO por ko-ru-to y PA por pa-ra-wa-jo, pero, por lo demás, contienen el mismo texto:

11 Ferluga, "La terminologia...", cit., p. 382.

12 Como quiere Ferluga, "La terminologia...", cit., p. 382, cf. DMic. s. v. Cf. Scafa, "L'epica...", cit., p. 60.

13 Quizá la misma que escribe Wa 731 y 732.

14 Cf. Melena, "Further...", cit., Palaima, "Contiguities...", cit. 
ARM 1 me-zo-a $2 O 20$ me-u-jo-a $O 10$ KO $O 4$ PA 2.

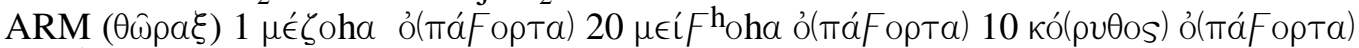
$4 \pi \alpha\left(\rho \alpha F^{h} \alpha i ́ \omega\right) 2$

1 Armadura: apliques grandes 20; apliques pequeños 10; apliques del casco 4; orejeras 2.

b) Un segundo grupo de cuatro tablillas ( $\mathrm{Sh} 734,735,738,739$, todas palimpsestos, esto es, reutilizadas) tienen un texto idéntico entre sí:

ARM 1 me-zo-a $2 O 22$ me-u-jo-a $O 212$ ko-ru-to $O 4 P A 2$.

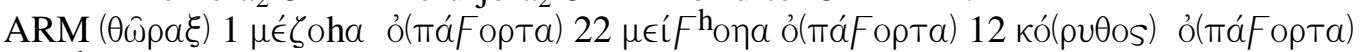
$4 \pi \alpha\left(\rho \alpha \mathrm{Fh}_{\alpha i ́} \omega\right) 2$

1 Armadura: apliques grandes 22; apliques pequeños 12; apliques del casco 4; orejeras 2

Vemos que el texto de las tablillas de este grupo solo se diferencia del de las del otro grupo en el número de o-pa-wo-ta $(O)$, ya que tienen dos más de cada tamaño: 22 grandes (en vez de 20) y 12 pequeños (en vez de 10).

Completan la serie dos tablillas diferentes de las demás:

En la primera de ellas, Sh 736, leemos:

to-ra-ke , a-me-ja-to , o-pa , me-za-na , wo-ke , ne-wo ZE 5

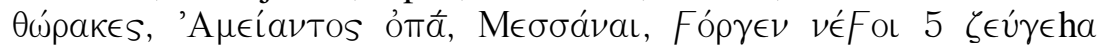

Armaduras nuevas, manufactura ${ }^{15}$ de Ameyante, fueron trabajadas en Mesana ${ }^{16}, 5$ pares.

Si wo-ke es un verbo ${ }^{17}$ es desde luego una forma de la raíz *werg- / wrg-, que da lugar a

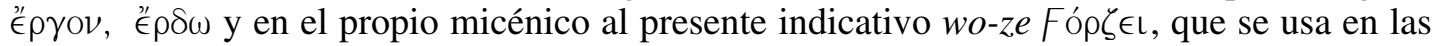
llamadas 'tablillas del catastro' (PY Ea 309, Eb 156.1a, 338 B, al.) para referirse a actividades que deben realizar determinados beneficiarios de entregas de grano, y al participio de pre-

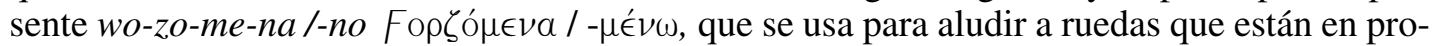
ceso de fabricación (KN So 4438, 4433.b) ${ }^{18}$. Aquí he preferido, aunque debo confesar que sin gran entusiasmo, aceptar la opción Fó $\rho \in \mathcal{~} \in, 3^{\mathrm{a}} \mathrm{pl}$. de un aoristo radical atemático pasivo sin aumento, por seguir el supuesto de que la tablilla está escrita en una sintaxis regular, lo que no me parece absolutamente seguro ${ }^{19}$.

15 Cf. Melena, "Further...", cit. Otras conclusiones en C. Consani, "Per l'interpretazione di miceneo o-pa", SSL 17, 1977, pp. 31-66. Según Killen "Mycenaean...", cit. se trata de un trabajo que implica reparación o acabado de un objeto ya existente.

16 Traduzco me-za-na como topónimo (cf. DMic. s.v.) Killen, "Mycenaean...", cit., p.330 n. 26 considera la posibilidad de ver un antropónimo $M \in \sigma \sigma \hat{\alpha} \nu$. que podría ser el fabricante de la armadura ("in parenthesis as it where"). Tal propuesta, sin embargo, parte de la idea de que se trata de una reparación, no de una armadura nueva, sobre lo cual, cf. $\S 3.3 .5$.

17 Una alternativa de interpretación sería la frase no tuviera verbo y me-za-na, wo-ke fuera todo ello un topónimo /Metsanās woikei/ 'en el woikos de M.', como quiere L. R. Palmer, "War and Society in a Mycenaean kingdom", Armées et fiscalité dans le monde antique, Paris 1977, pp. 35-64: 55.

18 Cf. DMic. s.v. wo-ke.

19 Cabe también aceptar $F o ́ p \zeta \in \mathrm{L}$ pres. ind., con una alternancia gráfica con la otra forma documentada wo$z e$, como la que hay entre $a-k e-t i-r i-j a$ y $a-z e-t i-r i-j a$, etc., con concordancia ad sensum: 'fabrica 5 pares nuevos'

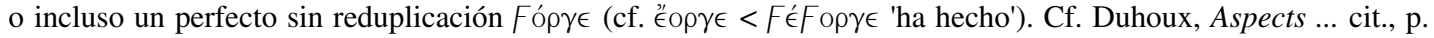
125. En todo caso, el detalle morfológico de esta palabra no constituye un problema para nuestro estudio sobre el vocabulario de las armas. 
La otra tablilla diferenciada, dentro de la serie (Sh 740) es paralela al grupo primero de la serie, pero con un añadido (la línea a) al principio del texto:

Sh 740

a

ko-ru-to $O 4 P A 2$

pa-ra-jo , ARM ZE 5 wi-so-ẉo-pa-țọ õ Õ o?-pa-wo-ta, me-zo-a ${ }_{2} O 20$ me-u-jo-a $O$

10.

La lectura wi-so-wo-pa-to es la aceptada por Bennet ${ }^{20}$, frente a wi-so-wo-pa-nq que había sido preferida hasta entonces y publicada en la edición del propio Bennet ${ }^{21}$. Dejando para más adelante la interpretación de este término concreto,? lo demás es claro:

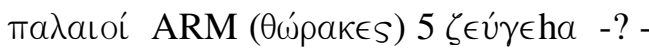

armaduras del año pasado: 5 pares -? -

Hay que entender pa-ra-ja como término propio de la administración de palacio en el sentido 'del año pasado', no como un concepto de estado 'viejo, usado'22.

Tenemos también la correspondiente etiqueta de la cesta en la que se contenía toda la serie, Wa 732 con la indicación de la palabra en plural, ] tọ-ra-ke.

\subsection{El vocabulario de la serie pilia $\mathrm{Sh}$}

\subsubsection{Armadura}

La mención de to-ra-ke en plural en 736 nos brinda la equivalencia del logograma ARM

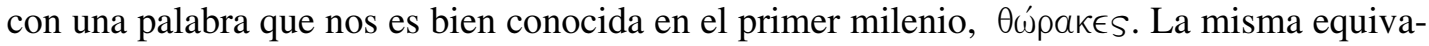
lencia se encuentra en Tirinte ( $\mathrm{Si}$ 5) en que hallamos por dos veces la forma singular to-ra$k a$ seguida del logograma ARM y del numeral uno. Dejamos el dossier de Cnoso para más adelante, porque presenta algunas novedades interesantes.

La mayor contribución para aclarar el estudio de esta palabra es una página que le dedica Killen ${ }^{23}$, quien pone de relieve que la palabra en micénico significa 'armadura' y no solo la 'coraza', como en el primer milenio. Por ello tanto en Pilo como en Cnoso a la mención de $\theta \hat{\omega} \rho \alpha \xi$ le siguen partes constitutivas tanto de la coraza como del casco. El logograma correspondiente (*163 ARM) dibuja sumariamente coraza, hombreras y casco. No extraña, en con-

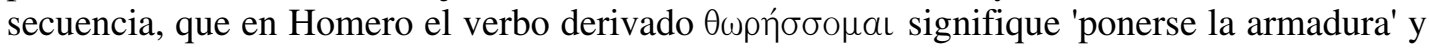
no 'ponerse la coraza', recogiendo el antiguo sentido de $\theta \omega \hat{\rho} a \xi$ como 'armadura'.

La palabra $\theta \omega \hat{\omega} \rho \xi \xi$ no tiene etimología conocida. Probablemente se trata de un préstamo ${ }^{24}$. En el primer milenio se especializa en el sentido de 'coraza', (incluso luego, por traslación, puede significar 'tórax' como parte del cuerpo) quedando el sentido primitivo más general, como hemos visto, sólo en los verbos $\theta \omega \rho \eta ́ \sigma \sigma o \mu a \iota$ (que conoce un curioso sentido traslaticio 'emborracharse') y $\theta \omega \rho a \kappa i \zeta \omega$.

20 Cf. E. L. Bennett Jr., "A selection of Pylos Tablets texts", en Olivier (ed.), Mycenaïka cit., pp. 103-127: 117.

21 E. L. Bennett Jr., J.-P. Olivier, The Pylos Tablets Trascribed, I Texts and Notes, Roma 1973.

22 Cf. Lejeune, MPhM I pp. 36 s, Bernabé et al. "Estudios...I", cit., p. 156.

23 Killen, "New readings...", cit. p. 30s. Cf. Palaima, "'Contiguities'...", cit., p. 382 n. 8.

24 Cf. teorías pelásgicas y crítica en D. A. Hester, "'Pelasgian'. A new Indo-European language?", Lingua 13, 1964-5, pp. 335-384: 354. 


\subsubsection{Casco}

El casco se designa con un término bien conocido del griego posterior, que en esta serie aparece en genitivo, bien escrito completo ko-ru-to (кópvӨos), bien en abreviatura acrofónica $K O$, nunca en forma de logograma, a diferencia de las referencias a cascos en Cnoso, que, como veremos, se encuentran en nominativo y van acompañadas de logograma. También está documentado una vez el instrum. ko-ru-pi-qe кópvтфí $\kappa^{\mathrm{W}} \epsilon$, en PY Ta 642.2 en referencia a una mesa decorada con otros temas 'y con (dibujos de) $\operatorname{cascos}^{25}$.

La etimología de esta palabra nos es desconocida. Chantraine ${ }^{26}$ niega, creo que con razón, la frecuente propuesta de relacionarla con képas 'cuerno'. Palabras relacionadas con

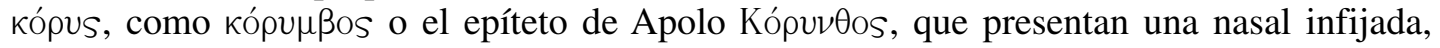
podrían apuntar a que se tratara de un término prehelénico. Incluso se ha barajado su posible origen pelásgico ${ }^{27}$.

En el griego alfabético kópus es una palabra de uso corriente, que conserva siempre el mismo sentido, y que da lugar a un amplio número de compuestos y derivados.

\subsubsection{Apliques de la armadura y del casco}

Tanto la armadura como el casco se componían de piezas, llamadas o-pa-wo-ta, una palabra que leemos, con pocas dudas, ómáFортa esto es, el neutro plural de un compuesto de

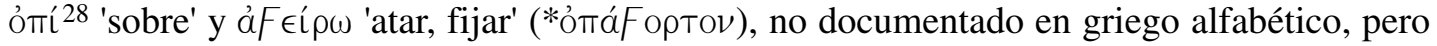
para el que se pueden ofrecer paralelos como ảopтń 'tahalí' o ả

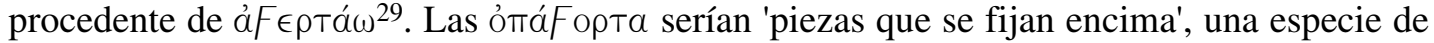
placas o apliques que se utilizan para la protección del coselete y el casco. Nunca se expresa el sustantivo con el que concertaría este adjetivo, por lo que, a falta de documentación en contra, resulta más económico entender que se trata de un adjetivo sustantivado ${ }^{30}$.

Aunque el segundo elemento del compuesto, el que da lugar a áF€í $\rho \omega$, carece de una etimología clara, es evidente que el compuesto ómáf opta se ha creado ya dentro del griego como una designación descriptiva, local, de las piezas en cuestión. El término no tiene continuidad en el primer milenio, probablemente porque dejan de fabricarse armaduras de este tipo, con piezas o apliques.

Para nuestro estudio carecen de interés las menciones de tamaño que las califican, formadas con el sufijo *yos- (con valor aún 'intensivo', antes de dar lugar a comparativos al unírseles el segundo término) me-zo- $a_{2}$, /medzoha/), <*meg-yos-a 'más (bien) grandes' y me-ujo- $a_{2}$, /meiwyohal < *meiw-yos-a 'más (bien) pequeñas'. El número de ambas, siempre par, es, según la proporción entre unas y otras, de 20 y 10, o de 22 y 12. Se ha discutido la forma en que se dispondrían estas 'placas' sobre una base que probablemente sería una pieza textil o de cuero ${ }^{31}$.

Aparecen, también, referidas al casco, 4 ótráFортa, lo que quiere decir que se emplea para los apliques del casco el mismo término que para los apliques de la armadura. El núme383.

25 Sobre nombres propios derivados de esta palabra en micénico cf. Ferluga, "La terminologia...", cit., p.

26 P. Chantraine, Dictionnaire étymologique de la langue grecque, París 1968 etc. , s. v.

27 Hester, "Pelasgian...", cit., p. 357.

28 Cf. A. Morpurgo Davies, "Mycenaean and Greek prepositions: o-pi, e-pi, etc.", en Heubeck y Neumann (ed.), Res Mycenaeae, cit., pp. 287-310: 298.

29 Docs. p. 376.

30 Cf. Shelmerdine, "From Mycenae...", cit. p. 476s.

31 Cf. Ruipérez y Melena, Los griegos..., cit., p. 205 un resumen de las diversas propuestas de disposición. 


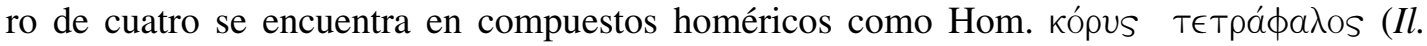

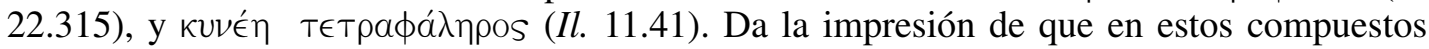
falov" ha tomado el lugar de ỏmáFорта.

\subsubsection{Orejeras}

El casco tiene, además, otras dos piezas, denominadas pa-ra-wa-jo, nominativo dual $\pi \alpha \rho \bar{\alpha} F h_{\alpha i ́} \omega$, esto es, 'las dos orejeras', piezas del casco que cubren la mejilla. Se trata de un adjetivo sustantivado, derivado en *-yo- de la palabra que designa la 'mejilla', $\pi \alpha \rho \bar{\alpha} F \mathrm{~h} \bar{\alpha}$, que es un compuesto de Trapá y de una variante del nombre de la 'oreja', aủ $\sigma^{-32}$.

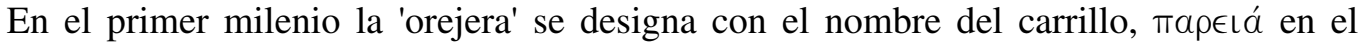
HHom. 31.11 y en diversas inscripciones, pero en Homero encontramos la forma con *-yo тари́ıov para designar la mejilla y una pieza del arnés que cubre el carrillo del caballo. Asi-

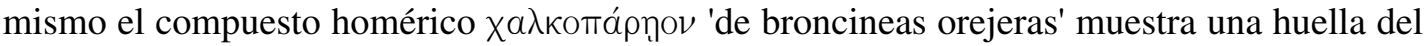
antiguo derivado en $*$-yo- para designar esta pieza de la armadura.

\subsection{5. wi-so-wo-pa-țo}

Nos queda la misteriosa forma wi-so-wo-pa-to (lectura alternativa de wi-so-wo-pa-na).

Sin entrar en aspectos paleográficos (que no son mi especialidad), estimo que es más esperable wi-so-wo-pa-tọ, que sería un nominativo plural concertado con $\theta \omega ́ p a \kappa \in S$ (encubierto bajo el logograma ARM), con el que también concierta pa-ra-wo, y no wi-so-wo-paṇa que tendría que concertar con o-pa-wo-ta en neutro plural, frente al hábito del escriba, que no califica nunca esta palabra en ninguna de las otras diez tablillas.

Recordemos que la palabra que nos ocupa se encuentra en la tablilla Sh 740 precedida de la mención 'cinco pares de armaduras viejas' y seguida de las indicaciones '20 apliques mayores y 10 menores, cuatro apliques del casco y dos orejeras'. Ello quiere decir que cada armadura estaba compuesta por el citado número de apliques y los componentes de un casco. $\mathrm{Si}$ esto es así, la palabra wi-so-wo-pa-tọ situada entre la designación de los cinco pares de armaduras y la serie de componentes de una armadura debe ser la manera de calificar las armaduras que tienen este número de piezas. En cambio, en Sh 736 ha desaparecido esta indicación, porque, como vemos, unas son de $20+10$ apliques y otras de $22+12$. Todo tendría mucho sentido si wi-so-wọ-pa-țo significa algo así como 'con el mismo número de apliques'.

Pasemos a examinar la forma de nuestra palabra misteriosa: parece ser un compuesto cuyo primer término es casi con seguridad $F\left\llcorner\sigma F O^{-}\right.$'de igual número de'. Para el segundo término, se han hecho diversas propuestas, que han sido criticadas por Del Freo ${ }^{33}$. Su propia pro-

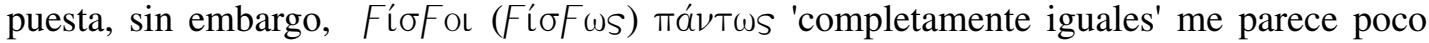
esperable desde el punto de vista semántico. Es rara una redundancia semejante en la econo-

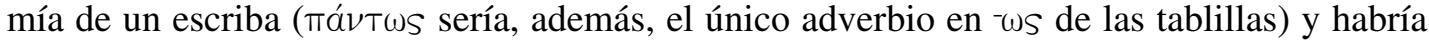
que suponer, además, que el escriba ha escrito como una palabra lo que son dos. Prefiero seguir con la hipótesis de que se trata de un compuesto, para el que propongo la solución que me parece más obvia y fácil y quizá, por ello, la más verosímil: que la palabra significaba lo que esperaríamos por su posición en la tablilla, esto es, 'con el mismo número de ỏmáf ортâ. Para ello sólo tendríamos que aceptar un leve error del escriba, que habría escrito wi-so-wo-

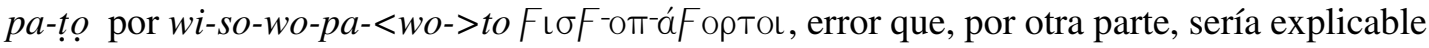

\footnotetext{
32 Cf. el detalle en Chantraine, Dict. étym. s. v.

${ }^{33}$ Cf. Del Freo, "wi-so-wo-pa-na ... ", cit.
} 
por la extrema longitud de la palabra y por la presencia de otra sílaba -wo- anterior, que podría haber provocado la haplografía. Si es así, el adjetivo 'del mismo número de apliques' sería explicado en la tablilla por la indicación posterior (a saber, 20 apliques mayores, 10 menores, etc.) e insistiría en que todas las armaduras del grupo reseñado tienen el mismo número de apliques, esto es $20+10$. Se explicaría así por qué en la tablilla Sh 736 no aparece el adjetivo, porque no serían todas del mismo número de apliques, sino que unas tendrían $20+10$ y otras $22+12$.

Killen ${ }^{34}$ argumenta de un modo parcialmente coincidente con el mío, pero prefiere leer dos palabras, /wiswoi panton/ '̌́ol má $\nu T \omega \nu$ esto es 'idénticas en todo' o mejor ambas en geni-

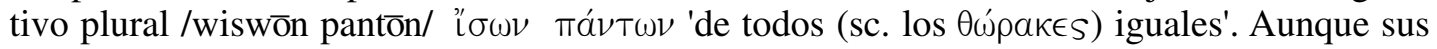
propuestas son más aceptables que la de Del Freo, pienso que en la primera de ellas el uso de $\pi a ́ v T \omega \nu$ seguiría siendo redundante, ya que, si las armaduras son 'idénticas' obviamente lo son 'en todo'. En cuanto a la segunda propuesta, me parece sintácticamente muy complicada. Se

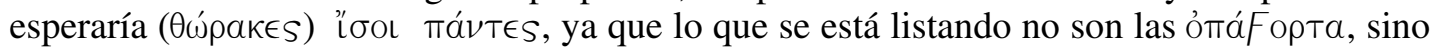
las corazas (ideograma ARM). Me resulta más natural pensar en una redacción 'cinco corazas de igual número de apliques, (esto es) veinte apliques grandes... etc.', que en una redacción más extraña, 'cinco corazas, de todas iguales veinte apliques grandes....' etc.

Ello nos lleva a interpretar la relación existente entre la tablilla Sh 736 y 740. Según la communis opinio $^{35}$, Sh 740 sería la fiche d'entrée recapitulativa de la situación de las armaduras antes de ser reparadas, mientras que Sh 736 sería la fiche de sortie que indicaría el estado final de las armaduras. Las otras de la serie describirían la situación de cada una de ellas por separado. Si es así, el numeral 5 tras el logograma ZE ('par') indicaría que se trata de diez armaduras (cinco pares) tanto en 736 como en 740. Diez armaduras que, al entrar en el taller, tendrían todas $20+10$ ỏ $\alpha \dot{F}$ ор ота, mientras que al salir, unas tendrían $20+10$ y otras $22+$ 12. Las indicaciones pa-ra-jo y ne-wo indicarían el estado anterior y posterior al hipotético arreglo. Aceptar esta hipótesis plantearía problemas adicionales: no sólo el par de términos no tendría aquí el sentido más habitual en la organización palaciega, en la que pa-ra-ja se refiere a '(entregas) del año anterior', y ne-wo a las 'del año en curso' ${ }^{36}$, sino que tendríamos que aceptar para ne-wo un significado 'reparado' (porque lo más que tendrían las armaduras es el añadido de un par de apliques, y eso no bastaría para considerarlas 'nuevas') y para $p a-$ ra-jo un valor de deterioro, asimismo difícil de admitir. O, aún más difícil, aceptar que se refiere a 'modelos antiguos' y 'modelos nuevos', como si las de más o-pa-wo-ta fueran las 'de moda' en el momento.

Otra posibilidad es que las fichas recapitulativas no se refirieran a la entrada y la salida de las mismas armaduras, sino a dos partidas de orígenes distintos ${ }^{37}$. En la tablilla $\mathrm{Sh} 740$ el escriba habría consignado una partida de armaduras del año anterior. Serían cinco, no diez armaduras, contadas por pares porque tienen parte delantera y trasera, de igual modo que en español puede hablarse de dos pares de gafas para hablar de dos gafas. Las cinco serían de $20+10$ öта́F орта. Por su parte, en Sh 736 habría asentado 5 armaduras del presente año, hechas por un artesano llamado Ameyante. De ellas cuatro serían de $22+12$ ómáFopta y una de $20+10$. La suma de las dos partidas, diez armaduras, coincide con las 10 de las tablillas individuales. La propuesta tendría la ventaja de que los valores de pa-ra-jo y ne-wo serían en estas tablillas los habituales, de carácter administrativo.

\footnotetext{
34 Killen, "New readings"... cit.

35 Cf. Vandenabeele-Olivier, Les idéogrammes ..., cit., pp. 22-24; Palaima, "Contiguities...", cit.; Killen, "Mycenaean ...", cit., p. 329.

36 Cf. M. Lejeune, MPhM I pp. 36s.; Bernabé et al., "Estudios...I", pp. 155s.;"Estudios...II", p. 136.

37 Cf. Melena, "Further thoughts...", cit., 276, quien cree que forman un díptico y eran las dos primeras del set, seguidas, probablemente por Sh 737.
} 
Sea como fuere, esta cuestión no incide directamente sobre la interpretación lexicográfica de los términos, que es la que aquí me preocupa.

\subsection{Armaduras en Cnoso}

Con respecto a las armaduras, el dossier de Cnoso nos brinda datos similares a los de Pilo, pero también algunas novedades. Hablo de la serie Sk, compuesta por seis tablillas (Sk $789,5670,7751+9195+, 8100,8149$ y 8254$)$, la mayoría de ellas en muy mal estado, si bien la $n^{\circ} 8100$ nos permite hacernos una idea de la disposición de los elementos:

.A

. Ba

$\mathrm{Bb}$

$$
\text { qe-ro } 22 \text { e-po-mi-jo } 2 \text { / o-pa-wo[-ta }
$$

o-pa-wo-ta 2

to-ra / ko-ru GAL 1 o-pi-ko-ru-si-ja 2 pa-ra-wa[-jo 2

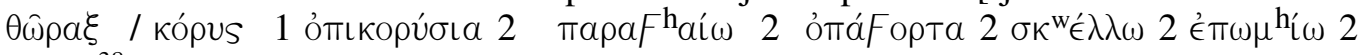
о̉тáFорта ${ }^{38}$

armadura: casco 1; piezas sobre el casco, 2; orejeras, 2; apliques, 2; coseletes, 2; hombreras, 2; apliques [

La armadura aparece mencionada por su nombre to-ra, que no es otra cosa sino una grafía alternativa para $\theta \omega \hat{\omega} \rho \xi \xi$, como señaló Killen ${ }^{39}$, apoyándose en paralelos de este tipo de alternancias como $o-n u$ frente a $o$-nu-ka como reflejo de ővv $\xi$. No va acompañada ni de logograma ni de numeral, pero la grafía to-ra y la presencia pa-ra-wa-jo en dual en 789 indica que se trata en cada caso de una armadura. Como en Pilo, siguen referencias a los componentes de la armadura. Aquí primero el casco, que no se menciona en genitivo ni con la abreviatura, como en Pilo, sino escrito silábicamente en nominativo ko-ru y seguido del logogra-

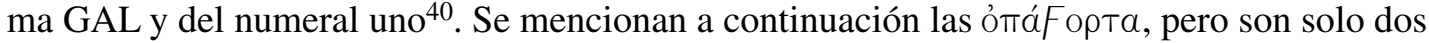
y no cuatro como en Pilo. En cambio se añaden dos piezas más, llamadas una vez (789) $e$ pi-ko-ru-si-jo en dual y dos veces $(8100,8149)$ o-pi-ko-ru-si-ja en plural. Probablemente no es casual que las dos veces va acompañado de la escritura, encima, de o-pa-wo-ta (también en plu.) seguido de 2 , mientras que en la tablilla en que aparece e-pi-ko-ru-si-jo no parece haber referencia a las o-pa-wo-ta, pero no acabo de entender la razón de este uso del escriba. Completa la relación de piezas del casco el par de $\pi \alpha \rho \alpha F^{h} \alpha i ́ \omega$ 'orejeras', como en Pilo.

También en la mención de la coraza encontramos algunas diferencias. En vez de las referencias a ómáf opтa mayores y menores, típicas del archivo pilio, encontramos la palabra qe$\mathrm{ro}_{2}$ 'coselete'.

En la serie Sk de Cnoso se añaden a la designación de las partes del coselete un par de piezas, también desconocidas en Pilo, los e-po-mi-jo $\epsilon^{\prime} \pi \omega \mu^{\mathrm{h}} \hat{\imath} \omega$ 'hombreras ${ }^{\prime 41}$.

$q e-\mathrm{ro}_{2}$ aparece documentada, fuera de aquí, una vez en plural (KN K 740), en una relación de objetos diversos (30 vasijas di-pa de bronce, un vaso, quizá con adornos en oro, y una sierra). Podría ser la relación de trabajos de un broncista:

\footnotetext{
38 En esta tablilla la segunda línea debe leerse en primer lugar y la primera, en segundo lugar.

39 Killen, "New readings...", cit., p. 30s.

40 Parece faltar en 789

41 Sobre la preferencia por esta grafía cf. A.Bernabé, "Towards a new interpretation of the Osthoff's Law", Historische Sprachforschung 103, 1990, pp. 220-235: 225 y 231.
} 


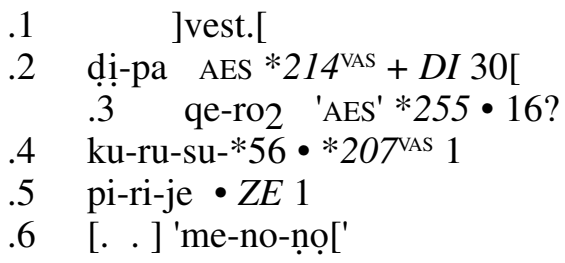

En esta tablilla acompaña, ademas, a qe-ro 2 el logograma $* 255$, que nos orienta sobre su forma, aproximadamente cuadrada.

\subsection{Vocabulario sobre armas defensivas específico de Cnoso}

\subsubsection{Apliques del casco}

Parece, pues, que en Cnoso el casco también estaba compuesto de cuatro piezas, como en Pilo, pero la diferencia es que dos de estas piezas reciben un nombre especial, en dos variantes, e-pi-ko-ru-si-jo y o-pi-ko-ru-si-ja, que se reconocen con claridad como є̇ாเКо-

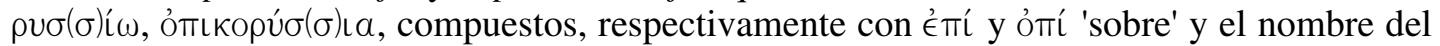
casco кópv $\theta^{-}$, provistos del sufijo de derivación $*_{-} y o^{42}$ (cf. para la formación émı $\theta a \lambda a ́ \sigma^{-}$ $\sigma(O S)^{43}$. La $\theta$ ha sufrido asibiliación ante ı, lo que es un rasgo sobradamente conocido del micénico. Es, pues, un típico compuesto que reposa sobre una determinación local que hace referencia a otro elemento más importante y conocido. La palabra no sobrevive a la época

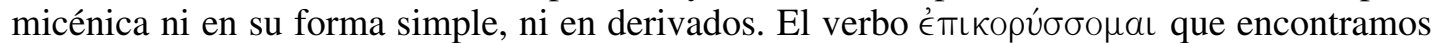
en Luciano ${ }^{44}$ es una innovación, con el sentido 'armarse de nuevo', con otro valor de étí completamente distinto del que tiene en micénico.

\subsubsection{Coselete}

Como hemos visto, en vez de las referencias a ỏmáFopta mayores y menores, típicas del archivo pilio, encontramos la palabra $q e-r_{2}$ 'coselete'. Acepto la propuesta de Heubeck $^{45}$

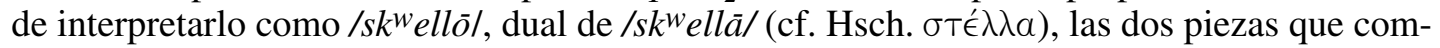
ponen un coselete bivalbo, realizado en cuero.

Según Heubeck *255 representaría la variante realizada en bronce, mientras que la pieza consignada en la serie Sk de Cnoso sería una variante confeccionada en cuero y con apliques metálicos (o-pa-wo-ta), cuya notación logográfica sería TUN $+Q E$, la forma en que la hallamos en KN Sc 224, 227, 229, etc. Su propuesta fue matizada por Del Freo ${ }^{46}$, quien cree que el logograma correspondiente para ambos tipos sería el $* 255$ (independientemente de la materia en que estuviera hecho), y no TUN $+Q E$. En opinión de Del Freo la oposición entre TUN $+Q E$ y el logograma $* 255$ radica en el tipo de objeto representado: TUN $+Q E$ se referiría a un tipo de armadura 'dotada de $q e-r o_{2}$ ', mientras $* 255$ trataría de los 'qe-ro $o_{2}$ tout court'.

Se inserta el término que reconstruimos como $s k^{w} e l l a-$ en una familia de palabras estudiada por Taillardat ${ }^{47}$, que, en palabras del autor, tienen en común el designar cualquier obje-

42 Cf. Morpurgo Davies, "Mycenaean...", cit., p. 295s., quien sostiene que probablemente se originan en una frase del tipo *opi koruthi, redeterminada con un sufijo *-yo-.

43 Sobre el tipo de compuesto cf. F. M. J. Waanders, "Compounds in Mycenaean Greek". en De Miro, Godart, Sacconi (edd.), Atti e Memorie... cit.,, pp. 272-277.

44 Luc. Alex. 57.

45 Heubeck, "Mykenisch qe-ro 2 ", cit., "Vermutungen...", cit., mejor que interpretarlo como un "brazal" (cf.

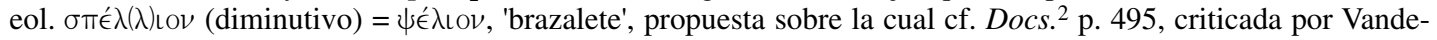
nabeele-Olivier, Les idéogrammes..., cit., p. 21).

46 Cf. Del Freo, "Mic. qe-ro $2 . . . "$, cit.

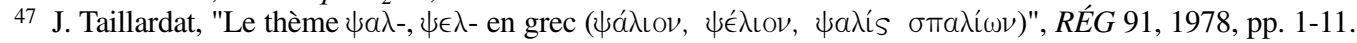


to en forma de círculo interrumpido, aunque la reconstruye $\sigma \pi \alpha \lambda-, \sigma \pi \epsilon \lambda$-, eventualmente resueltos como $\psi a \lambda^{-} \psi \lambda^{-}$. Los testimonios micénicos, escritos con el signo qe apuntan a que éstos, a su vez, proceden de $* s k^{w} a l$-, $s k^{w} e l$-, con labiovelar ${ }^{48}$. En el segundo milenio encontramos deri-

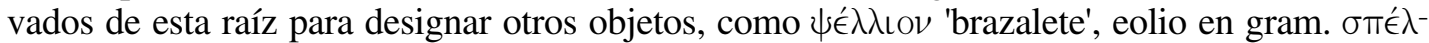

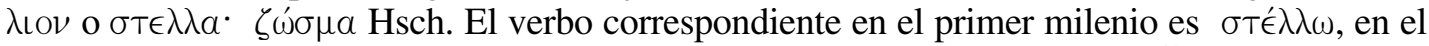
que ha podido producirse una confusión entre el tema $* s k^{w} e l$ - y otro ${ }^{*}$ stel-49, y que, en todo caso, ha evolucionado hacia un sentido muy general 'disponer, preparar, proveer de armas', etc.

\subsubsection{Hombreras}

En la serie Sk de Cnoso se añaden a la designación de las partes del coselete un par de

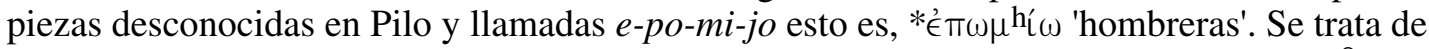
un adjetivo en *-yo- compuesto de ÉTí y del nombre de la parte del cuerpo que cubre, $\stackrel{\nexists}{\omega} \mu \mathrm{s}$, e. d. 'que va sobre el hombro'50. Esta ultima palabra es una designación de parte del cuerpo de antigua raigambre indoeuropea, cf. lat. humerus, etc. No sabemos si el término está sus-

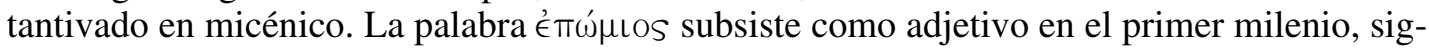
nificando, en general, 'que está sobre el hombro', y aplicado a diversas realidades. En el sentido 'hombrera' tiende a verse sustituido por ém

Una sola vez (Sk 8100) se repite o-pa-wo-ta, indicando quizá que hay ómáfopta de la coraza, pero lamentablemente se ha perdido el numeral. La disposición de los términos en esta tablilla no es, sin embargo, nada clara.

\subsection{Dos desconocidos: el escudo y las grebas}

Desconocemos la terminología del escudo, ya que no tenemos ningún documento en ninguno de los archivos que haga referencia a esta arma. En un trabajo anterior he sostenido la posibilidad de que el conocido escudo 'en forma de ocho' pudiera denominarse a-pi-qo-to

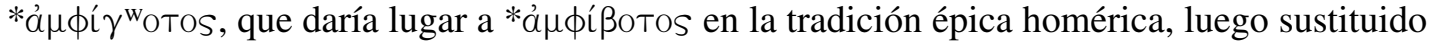

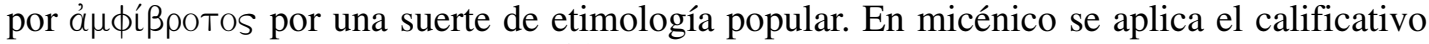
a-pi-qo-to a mesas y a una є́ $\sigma \chi \alpha ́ \rho \alpha^{51}$.

El armamento del guerrero micénico se completaba, de acuerdo con la representación del famoso 'vaso de los guerreros', con grebas o espinilleras. Pero también desconocemos la designación micénica de esta parte de la armadura.

\section{ARMAS OFENSIVAS}

\subsection{El arco}

No hay en las tablillas atestiguación del nombre del arco, pero parece que tal arma podría estar representada en el logograma $* 265$. Se encuentra este logograma en 3 tablillas, en bastante mal estado, halladas en la habitación de las tablillas de los carros de Cnoso:

48 Encontramos probablemente la misma raíz en el antropónimo qe-ri-jo $\Sigma^{\omega} \in \lambda \lambda$ ıós (correspondiente en

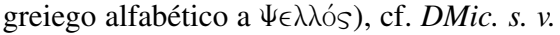

$49 \mathrm{La}$ forma mic. qe-ro 2 suministra la prueba de que el tema $*_{s} k^{w} e l$ - no es una 'vue de l'esprit' como sospechaba Chantraine, Dict. Étym. s.v.

50 Cf. Morpurgo Davies, "Mycenaean...", cit., p. 295s., quien propone un origen a partir de una frase del

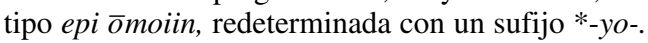

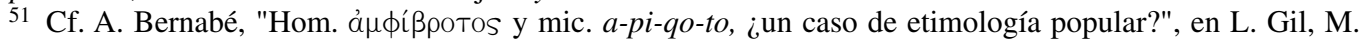
Martínez Pastor y R. M. Aguilar (eds.), Corolla Complutensis in memoriam J. S. Lasso de la Vega contexta, Madrid, Editorial Complutense, 1998, pp. 39-48. 


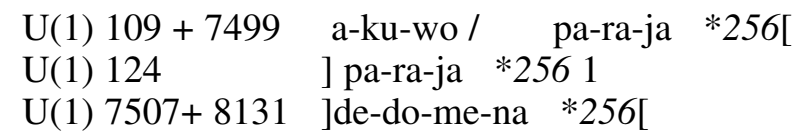

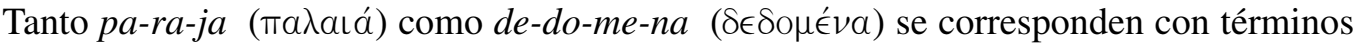
de la burocracia micénica que se relacionan con la distribución de trabajo a los artesanos por parte del palacio y del compromiso de éstos de entregarlo en un tiempo concreto: $\delta \in \delta o \mu \in ́ v O S$

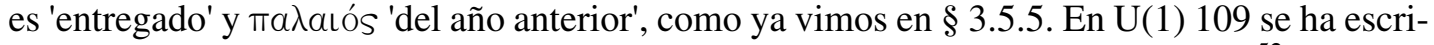
to pa-ra-ja encima de un de-tachado, probablemente comienzo de de-do-me-na ${ }^{52}$. a-ku-wo es un antropónimo.

Vandenabeele y Olivier ${ }^{53}$ no se pronuncian sobre la identidad del objeto, limitándose a señalarlo como uno entre tantos 'objects en comptabilité', y a considerarlo 'probablement du féminin'. La misma observación en cuanto al género hacen Duhoux ${ }^{54}$ y Ruipérez y Mele$\mathrm{na}^{55}$.

Mi propuesta es que el nombre del arco, correspondiente al logograma *256 es el neutro plural Tó $\xi a$. Las razones en que la apoyo son las siguientes:

a) Existen en micénico dos derivados de esta palabra, el 'arquero', to-ko-so-ta e. e. To Só- $^{-}$ $\tau \bar{\alpha} s$ en Cnoso (KN V(1) $150+7624)$ y el 'fabricante de arcos', to-ko-so-wo-ko, e. e. $T o \xi \mathrm{o} F \mathrm{op}^{-}$ yós en Pilo (An 207.12).

b) No está documentada en griego ninguna palabra femenina para el arco.

c) Sí lo está, en cambio, y abundantemente el plurale tantum Tó $\xi \alpha$ ya desde Homero, en que se usa Tó $\xi a$ concertando con adjetivos en neutro plural para designar un solo arco, e. g.

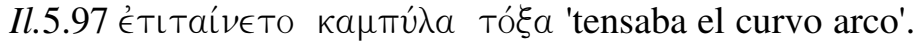

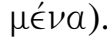

d) pa-ra-ja y de-do-me-na pueden ser neutros plurales (respectivamente maגaıá, $\delta \in \delta o^{-}$

e) La afirmación hasta ahora habitual de que el objeto tiene que ser femenino se basa en un presupuesto erróneo: como en U(1) 124 la palabra $p a-r a-j a$ va seguida del numeral 1 , se infiere que por ello necesariamente tiene que corresponder a una palabra en número singular. La afirmación no tiene que ser cierta si se trata de un plurale tantum, ya que en ese caso, aunque se refiera a un solo objeto, la palabra presentará morfológicamente siempre una forma plural y los adjetivos o participios que concierten con ella irán asimismo en plural, pero si tienen que llevar un numeral, éste será 1 . Baste señalar paralelos corrientes en español como unos pantalones viejos o unas tijeras rotas, refiriéndose en ambos casos a una unidad.

En consecuencia, entiendo que lo más económico es considerar que la palabra micénica

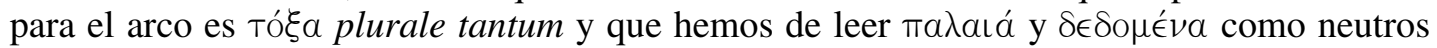
plurales, pero referidos (al menos en el caso de pa-ra-ja en que tenemos el numeral uno) a un solo arco.

En cuanto a su formación, Tó $\xi a$ parece ser un préstamo iranio (cf. persa taxš-), quizá

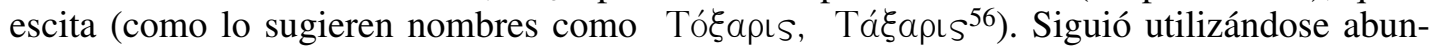
dantemente, en plural o en singular, en el griego del primer milenio.

\footnotetext{
52 Cf. $K T \mathrm{~V}$ "pa- over [[de]] (cf. ]de-do-me-na *256[ in U 7507)".

53 Vandenabeele - Olivier, Les idéogrammes ..., cit., p. 299.

54 Duhoux, Aspects ... cit., p. 124.

55 Ruipérez - Melena, Los griegos..., cit., p. 207.

56 Cf. Chantraine, Dict. étym. s. v. Tó $\xi_{0 \nu}$ y Scafa, "L'epica...", cit., p. 60.
} 


\subsection{Otras armas arrojadizas (PY Jn 829.3)}

Una línea de una tablilla de Pilo (PY Jn 829.3) nos documenta que se ha librado una cantidad de bronce procedente del templo (o quizá de un barco) ${ }^{57}$ para fabricar puntas de dos armas arrojadizas:

ka-ko , na-wi-jo ,pa-ta-jo-i-qe ,e-ke-si-qe ,a a-ka-sa-ma

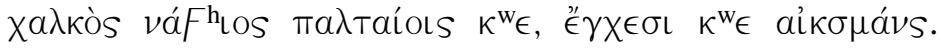

La línea en cuestión nos ofrece abundante información lexicográfica, pertinente para nuestro estudio. La aprovecharemos, desglosada, en los parágrafos siguientes.

\subsection{Puntas}

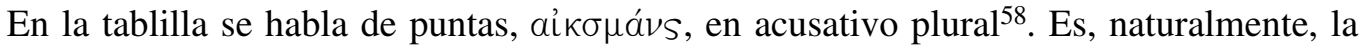
punta lo que es de bronce en estas armas. Dado que es la parte más significativa y relevante de la lanza no extraña que en el primer milenio, aỉx $\mu$ ń, además de seguir usándose con su sentido 'punta' sirva para designar la 'lanza', por una conocida evolución de pars pro toto ${ }^{59}$.

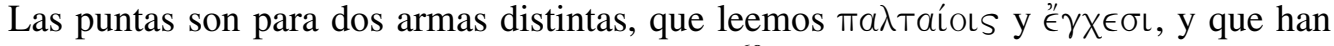
sido interpretados como dativos directivos por Brixhe ${ }^{60}$.

\subsection{Lanza}

e-ke-si documenta para el micénico el mismo término é $\gamma \chi X S$ que es usual en Homero para designar la lanza, cf. por ej. Il. 6.319-20 en que Héctor lleva una lanza de once codos, que también va provista de una punta de bronce llamada ai $x \mu \eta$ :

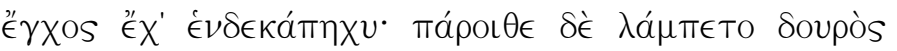

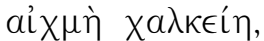

'Llevaba una lanza de once codos. Por delante del asta relucía la punta broncínea'.

Volvemos a encontrar el término, en nominativo del plural en KN R 1815:

e-]ke-a / ka-ka re-a? HAS 12.

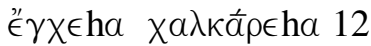

'12 lanzas guarnecidas de bronce',

un sintagma del que también hallamos paralelos homéricos, como por ejemplo, la fórmula $\chi a \lambda \kappa \eta ́ \rho \in \sigma \iota \nu ~ \in ́ \gamma \chi \in i ́ n \sigma \iota \nu$ 'lanzas guarnecidas de bronce' (Il.18.534 etc.).

57 Puede tratarse de $v a ́ F^{\mathrm{h}}$ Los un derivado de la palabra para el templo (gr. $\left.v \bar{a} o ́ s, v \in \omega ́ s\right)$, o de $v a ́ F$ los, derivado del nombre de la nave (gr. vaûs).

58 Forma antecesora de la del primer milenio aixuás, con la desinencia de acusativo aún en -ns, y antes de que la $*_{-s-}$ entre $* k$ y $* m$ se haya perdido, aspirando la oclusiva anterior. La palabra es de origen indoeuropeo, relacionada con la serie de ákń, ảkís, lat. acus, etc. Cf. A. Bernabé, "Resultados en griego de las raíces con dos laringales (tipo HEH-)", RSEL 5, 1975, pp. 345-381.

59 Cf. Panagl, "Mykenisch...", cit., p. 506.

60 C. Brixhe, "Du 'dativ' mycénien aux protagonistes de la signation linguistique", en Olivier (ed.), Mykenaïka cit., pp. 129-157: 137 n. 44; 142. 
Es dudoso si hemos de leer el instrumental plural de esta misma palabra en la forma fragmentaria ]e-ke-pi [ en KN Wb 5131.1 ${ }^{61}$. En cambio parece claro que $e$-ko en $\mathrm{KN} \mathrm{Sc} 226$, donde aparece, seguido del numeral 1, tras un antropónimo y los logogramas del carro con ruedas, la armadura y el caballo, también seguido del numeral 1, debe interpretarse como el participio É $\chi \omega \nu$. A un individuo se le asigna un solo caballo, en vez de dos, 'porque tiene' otro $^{62}$.

Como otros nombres de armas en griego, $\epsilon^{\prime} \gamma \chi 0 S$ carece de etimología y, en opinión de Chantraine ${ }^{63}$, podría ser un préstamo. En el primer milenio se ha conservado, aunque de

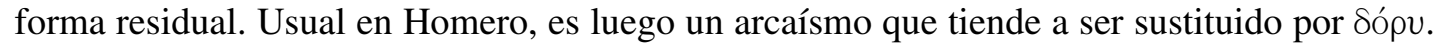
Se usa a veces como 'arma' en general (Pi. Pi. 9.28) y puede valer incluso por 'espada' (S.Ai. 287).

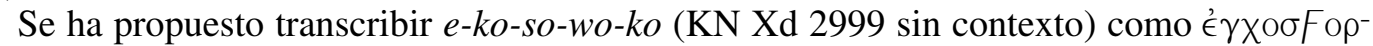

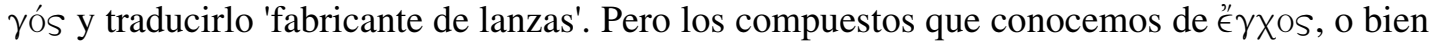

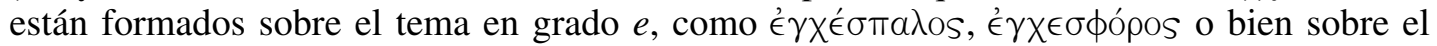

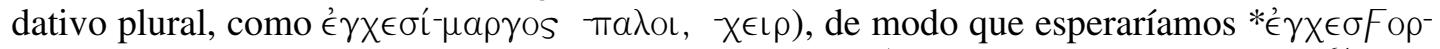

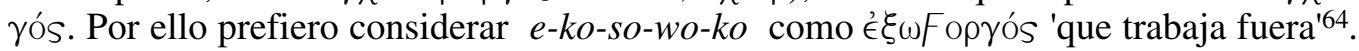

\subsection{Jabalinas}

Por su parte, $p a-t a-j a * \pi a \lambda$ тaîa son probablemente 'jabalinas' (aunque hay algunos problemas de identificación) ${ }^{65}$. La palabra reaparece en nom. plu. en tres nódulos de la serie Ws de Cnoso hallados en el Arsenal:

KN Ws 1704

.a JAC supra sigillum

.b o-pa

.g pa-ta-ja
KN Ws 8495

JAC supra sigillum

.b1 pa-ta-ja

.b2 vacat

.g $o-p a$
KN Ws 1705

sigillum

.b pa-ta-ja

.g vacat

La indicación o-pa (ỏmá) es de nuevo un término administrativo 'manufactura' ${ }^{66}$. En

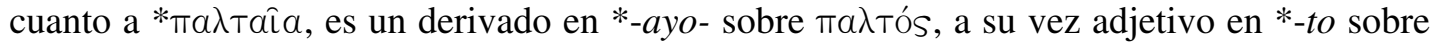
la raíz que da lugar al verbo má $\lambda \lambda \omega$ 'blandir', un verbo emparentado probablemente con lat. pello. En este caso no se trata de un préstamo, sino de un antiguo adjetivo que identifica el arma por su función, la de ser blandida y arrojada. La palabra correspondiente del primer milenio es та入тóv referida a cualquier arma arrojadiza (incluso a proyectiles de la catapulta).

Por otra parte, la palabra åk $\omega \nu$, bien conocida en griego desde Homero para designar la

61 Sobre nombres propios micénicos posiblemente derivados de esta palabra cf. Ferluga, "La terminologia...", cit., p. 383.

62 J. Driessen,"'Collecto items'. Observations sur l'élite mycénienne de Cnosos", en Olivier (ed.), Mikenaï$k a$ cit., pp. 196=214: 201.

63 Chantraine, Dict. étym. s.v.

64 Bibliografía en DMic. s.v.

65 Sobre las posibilidades de que este término designe a la 'jabalina' o la 'flecha' cf. Vandenabeele - Olivier, Les idéogrammes, cit., p. 59: a favor de que *254 (JAC) designe la jabalina está el hecho de su nombre (pa-ta-ja), así como el dibujo diferente de éste respecto a $* 230$ (HAS), por una parte, y*231 (SAG), ya que *254 no representa las "barbas" de la flecha, por otra. Figura en contra, el lugar de hallazgo (las "etiquetas" que llevan este ideograma aparecen en la "Armería", juntamente con los restos carbonizados de dos cajas de madera que contenían varillas carbonizadas y puntas de flecha, que argumenta a favor de la significación 'flecha' para *254).

${ }^{66}$ Cf. $§ 3.2$. 
'jabalina' y también de origen indoeuropeo (raíz *ak-, como la ya vista aỉk $\sigma \mu a ́ v s$ ), debía de haber sido conocida en micénico; aunque no aparece en nuestros documentos, está en la base

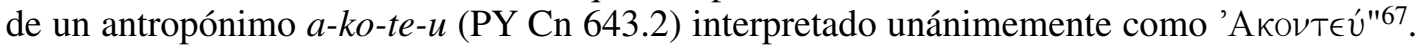

\subsection{Flechas}

Carecemos sin embargo de información sobre el nombre de las flechas, que aparecen siempre en la forma del logograma SAG. Se ha propuesto que podrían ser flechas las armas

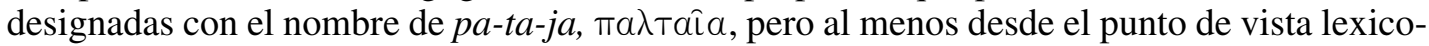
gráfico es más verosímil mantener la interpretación de esta última palabra como 'jabalinas'.

Una posibilidad podría ser que el nombre fuera $\gamma^{\mathrm{w} \epsilon ́} \lambda \in \mu \nu \alpha$ (cf. Hom. etc. $\beta \epsilon ́ \lambda \epsilon \mu \nu \alpha$ ), si hemos de leer el antropónimo qe-re-me-ne-u (PY Jn 845.13) como $\Gamma^{\mathrm{w}} \in \lambda \in \mu \nu \in u^{6}{ }^{68}$. Se trataría de un de derivado cuasi participial en - $m n$ - sobre el grado $e$ del radical que da lugar al verbo $\beta a ́ \lambda \lambda \omega$, asimismo de origen indoeuropeo ${ }^{69}$. Obviamente caben otras posibilidades ${ }^{70}$.

Aún podría añadirse el término iós si realmente forma parte del antropónimo $e$-ki-wo

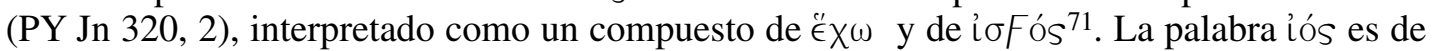
origen indoeuropeo (P. p. 301, 1*eis-, cf. ai. ișu-, av. išu- 'flecha').

\subsection{Una espada y una doble hacha en Ta 716}

En la serie Ta de Pilo, que parece ser un inventario del mobiliario y menaje de una sala de banquete, una de las tablillas, Ta 716 presenta objetos que parecen relacionados con la parafernalia que acompaña al propio rey en actos de representación ${ }^{72}$ :

.1 pa-sa-ro , ku-ru-so , a-pi , to-ni-jo 2 wa-o $* 2322$

.2 qi-si-pe-e $* 2342$

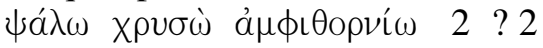

$\kappa^{\mathrm{w}} \sigma i \phi \in \mathrm{h} \in 2$

Se mencionan, en primer lugar, dos cadenas de oro para disponer a ambos lados del trono. Luego se asientan dos objetos llamados wa-o que (a juzgar por el logograma) podrían ser dobles hachas, el característico símbolo más heráldico y religioso que marcial y utilitario, que aparece representado múltiples veces en el mundo minoico. wa-o no parece tener un correlato conocido en el vocabulario griego (ya que no tiene nada que ver con hom. ăop, 'espada'), lo que nos hace pensar que probablemente se trata de un término minoico, cuyo nombre fue luego olvidado.

En la segunda línea se hace referencia a dos espadas ${ }^{73}$, acompañadas por el logograma *234, muy sumario y en una parte dañada de la tablilla, que no nos permite saber demasiado sobre su forma. El término que las designa qi-si-pe-e, es un dual de la palabra que en Home-

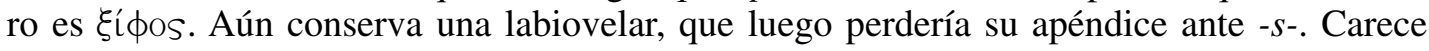

67 Cf. Ferluga, "La terminologia...", cit., p. 382, DMic. s. v.

68 Cf. Ferluga, "La terminologia...", cit., p. 382

69 Cf. Chantraine, Dict. Étym. s. v.

70 Cf. DMic. s. v.

71 Cf. A. Heubeck, "Bemerkungen zu den mykenischen Personennamen IV", BN 11, 1960, pp. 1-4: 3; C. J.

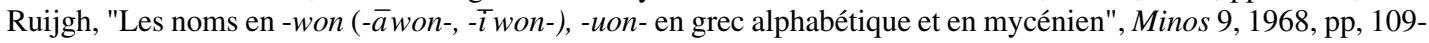
155: 148; Ferluga, "La terminologia...", cit., p. 382.

72 No comparto los puntos de vista de M. Del Freo, "Miceneo a-pi-to-ni-jo e la serie Ta di Pilo", SMEA 28, 1990, pp. 287-331.

${ }_{73}$ Un estudio arqueológico sobre diversos tipos de espada de los siglos XV al XII a.C. es el de N. K. Sandars, "Later Aegean bronze swords". AJA 67, 1963, pp. 117-153. 
también de etimología aceptable, ya que la hipótesis de un préstamo egipcio, que tuvo en tiempos cierto predicamento, parece hoy haberse derrumbado ${ }^{74}$.

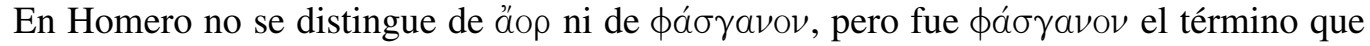
pervivió hasta el griego moderno.

\subsection{Espadas y dagas en el corredor de las tablillas de las espadas}

Más información lexicográfica sobre las armas micénicas puede encontrarse en la serie Ra de Knoso, hallada en el llamado 'corredor de las tablillas de las espadas' del palacio de Cnoso, en el que también se encontraron fragmentos de espadas ${ }^{75}$. En general el estado de las tablillas de la serie no es muy bueno. Dado, además, que hay notables oscuridades sobre la interpretación de algunos términos, conviene que comencemos por establecer cuáles son los ítems que interesaron al escriba. Hay dos grupos diferentes, denominados $\operatorname{Ra}(1)$ y $R a(2)$. Las tablillas $\mathrm{Ra}(1)$ se encontraron en el llamado "corredor de las tablillas de espadas", junto con fragmentos de espadas ${ }^{76}$, y todas ellas fueron escritas por la mano 126. Las tablillas $\mathrm{Ra}(2)$ aparecieron en el "pasillo de la Entrada Norte"77. Fueron escritas por la mano 127, que era también responsable de la serie Se, un inventario de CUR en el llamado "Bureau 2"78.

Las tablillas escritas por la mano 126, esto es $\mathrm{Ra}$ (1) deben distinguirse de las escritas por la mano 127, esto es, $\mathrm{Ra}(2)$.

El cuadro I muestra qué items se asientan en cada tablilla, siguiendo la siguiente disposición: 1) número de la tablilla; 2) nombre del artesano; 3) oficio; 4) arma; 5) apelativo que define el arma; 6) material; 7) presencia o no de de-de-me-na; 8) presencia o no de a-ra-ru-wo-a; 9) presencia o no de de-so-mo; y 10) logograma y cantidad, y 11) mano. El guión (-) precisa que el ítem ha sido omitido, mientras que el punto de interrogación (?) señala que desconocemos si el ítem figuraba o no, pues, si estaba, figuraría en una parte perdida de la tablilla.

Comenzamos por las tabllillas Ra (1). Descontamos como irrelevante para nuestros propósitos la aparición de to-sa en 1540, debida a que se trata de una tablilla totalizadora, con la acostumbrada indicación Tó $\sigma \sigma \alpha$ 'total'.

El escriba anota el número de $p a-k a-n a$ entregadas por cada artesano; puede escribir $p a-$

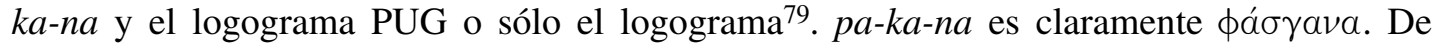

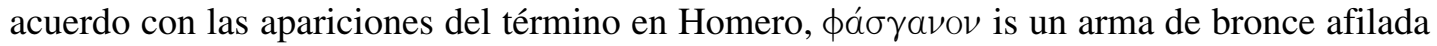

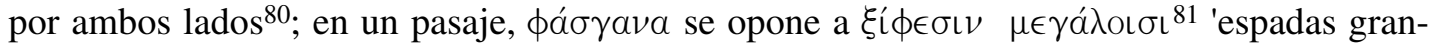
des'. Su sentido debe ser, pues, 'daga, espada corta' ${ }^{82}$. Se discute si se trata de un término de etimología indoeuropea (*sphag-skanon o *sphag-anon lit. 'cuchillo para el sacrificio') o semítica $(p s g)^{83}$. En cualquier caso es éste el término que pervivió en griego como designación general de la espada, incluso, como he dicho, hasta el griego moderno.

74 Cf. Chantraine, Dict. étym., s.v.

75 Sobre el corredor, cf. Hiller, "The 'corridor..." cit. Cf. asimismo A. Bernabé, "Some Thoughts on the Knossos Ra Series", en prensa.

76 Cf. S. Hiller, "The 'corridor...".

77 Cf. J. Driessen, "The Northern Entrance Passage at Knossos", in S. Deger-Jalkotzky - S. Hiller - O. Panagl (eds.), Floreant Studia Mycenaea (10. Mykenologisches Kolloquium. 30. April-6. Mai 1995. Salzburg), Wien, 205-226.

78 Cf. M. Lejeune,"Chars et roues à Cnossos: structure d'un inventaire", Minos 9, 1968, 9-61; A. Bernabé et al., "Estudios ... II".

79 Cf. 1543, 1546, and 1556.

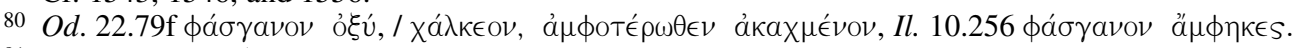

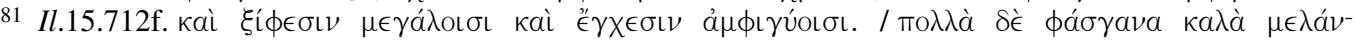

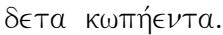

82 Cf. Vandenabeele-Olivier, Les idéogrammes ..., cit., 49ss.

83 Cf. Chantraine, Dict. étym. s. v. 
芒 $\quad$ do

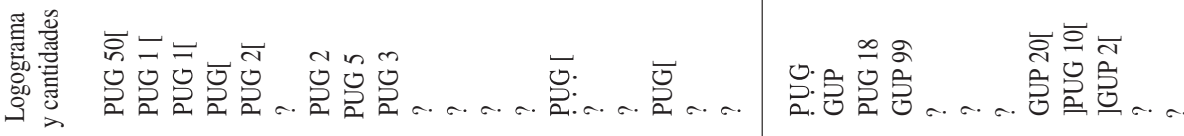

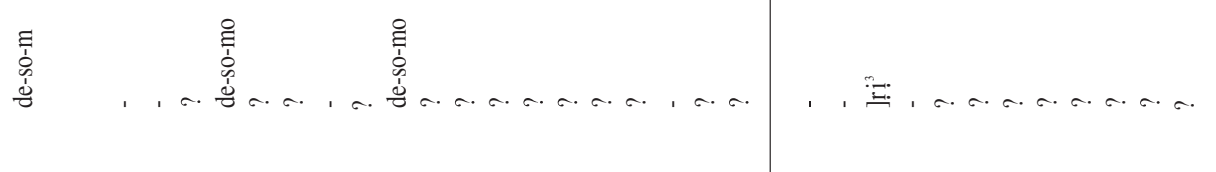

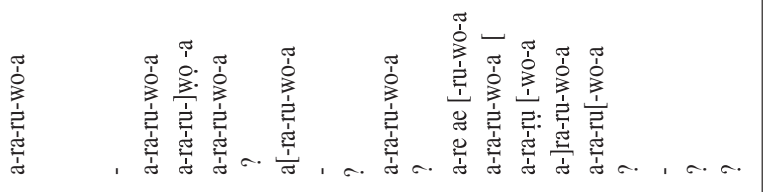

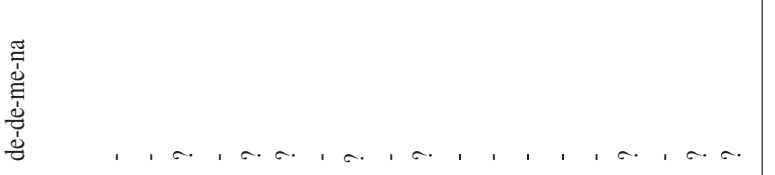

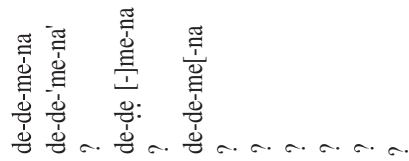
急
惡

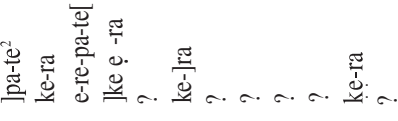

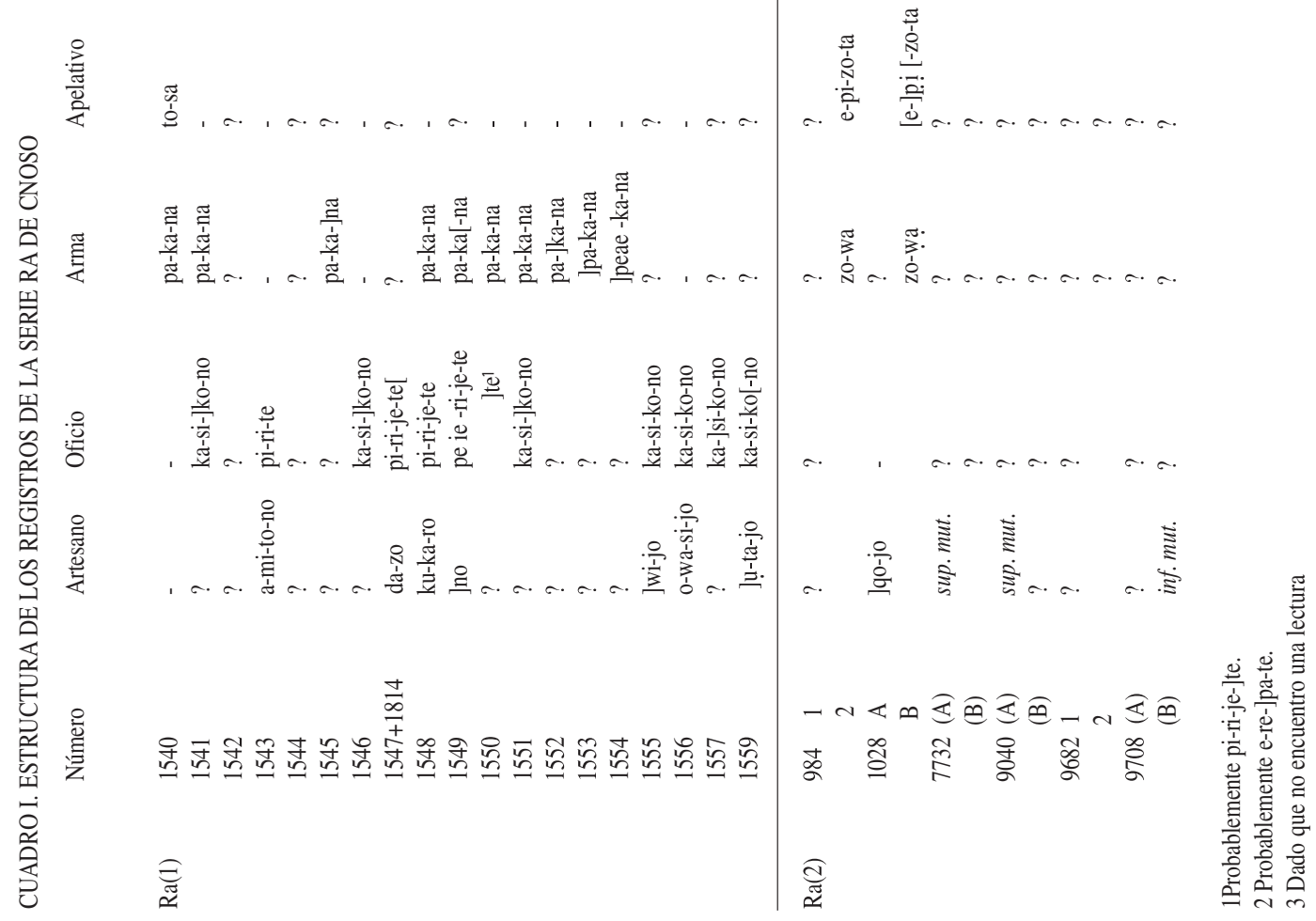


Las фá $\sigma \gamma a v a$, tanto las entregadas por un ka-si-ko-no como las entregadas por un pi-ri-je-

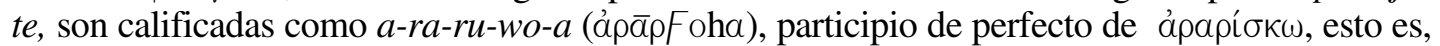
"ajustadas". De acuerdo con Palmer ${ }^{84} a-r a-r u$-wo-a 'debe tomarse absolutamente y la expresión puede referirse a la construcción técnica de la espada'; podemos entender que se trata de una espada con empuñadura sujeta con remaches. El perfecto insiste en el carácter de resultado, de obra acabada. El escriba registra que el trabajo está acabado. Como $a$-ra-ru-wo- $a$ se omite en 1546 y 1556, es probable que en estos dos casos la daga no estuviera acabada de montar.

En algunos casos leemos también de-so-mo, claramente un dativo de plural $\delta \in \sigma \mu o \hat{i} S^{85}$. Sobre el significado de $\delta \in \sigma \mu o \hat{s}$ se han hecho diversas propuestas ${ }^{86:}$ "un tipo de tahalí" (Gk. $\tau \in \lambda \alpha \mu(\dot{v})$ ), "remaches", o "empuñaduras". Encuentro más convincente la última propuesta ${ }^{87}$,

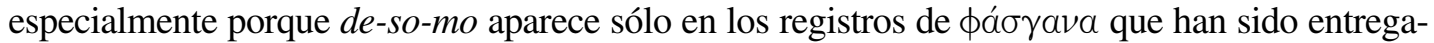
dos por un pi-ri-je-te y falta cuando el artesano es un ka-si-kono ${ }^{88}$. Aunque el material de las empuñaduras no se indica, podemos suponer que es marfil si comparamos estas tablillas con las de $\operatorname{Ra}(2)$ (cf. infra).

El escriba de Ra (1) indica siempre el oficio, así que debe considerar que esta información es importante. En cinco tablillas ${ }^{89}$ el oficio se denomina pi-ri-je-te con la variante pi-ri-te (en un caso $^{90}$, sólo puede leerse la última sílaba). Es evidente que el oficio del pi-ri-je-te está relaciona-

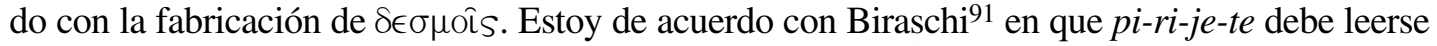

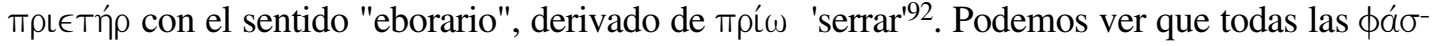

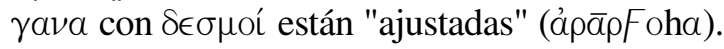

La interpretación de ka-si-ko-no es más difícil ${ }^{93}$. Hiller ${ }^{94}$ propuso con dudas la traducción "artesanos del cuerno", basándose en la tablilla KN V 684:

$$
\begin{array}{ll}
.1 & \text { e-re-pa-to / ka-ra-ma-to } 46 \\
.2 & \text { ka-so , ke-ma-ta } 8
\end{array}
$$

De acuerdo con Hiller, podemos suponer que " $k a$ - representaría un tipo de sinónimo (Minoico?) de кÉ $\rho$ ". . Es conjetural pero podría ser posible. Pero en la página siguiente ${ }^{95}$ relaciona piri-je-te con e-re-pa-te de-de-me-na y ka-si-ko-no con ke-ra de-de-me-na, relación que no coincide con lo que leemos en las tablillas $\mathrm{Ra}(2)$. De acuerdo con el Cuadro I pi-ri-je-te está claramente relacionado con pa-ka-na y de-so-mo, mientras ka-si-ko-no lo está con pa-ka-na sin de-so-mo y con los objetos designados por el logograma PUG, nunca objetos registrados como ke-ra de-de$m e-n a$, que siempre es solidario del logograma GUP.

Por otra parte, $k a-s i-k o-n o$ no se relaciona con una palabra que indique un material con-

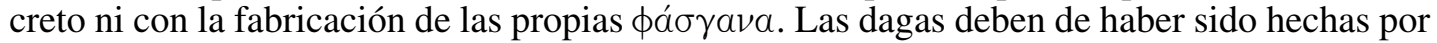

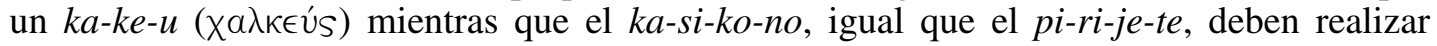

84 Cf. L. R. Palmer, The interpretation ...cit., 336.

85 C. Brixhe, "Du 'dativ' mycénien ...", cit., 137.

86 Cf. DMic. 167.

87 Cf. A. M. Biraschi, "Miceneo pi-ri-je-te: intagliatore d'avorio", PP 181, 1978, 281-287: 285; Hiller, "The 'corridor..." cit., p. 305, Ruipérez y Melena, Los griegos..., cit., p. 207.

881541,1546 y1556.

$891543,1547,1548,1549,1550$.

901550.

91 Cf. Biraschi, "Miceneo pi-ri-je-te:..." cit.

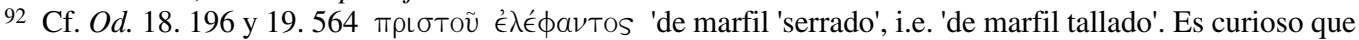

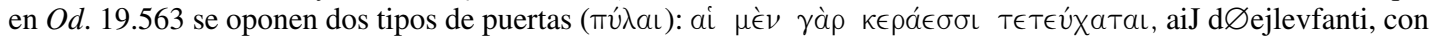
los mismos materiales usados para las armas en la serie Ra.

93 Cf. 'fonditore di stagno' según L. A. Stella, La civiltà micenea nei documenti contemporanei, Roma 1965 , 196 n. 9, o 'compagnon' según Lejeune, MPhM II, 248s.

94 Hiller, "The 'corridor..." cit., p. 305.

95 Hiller, "The 'corridor..." cit., p. 306. 
determinado trabajo subsecuente en ellas. Sugiero que el ka-si-ko-no era responsable de la incrustación de otros materiales en la hoja. Se han encontrado piezas excelentes que tienen incrustación

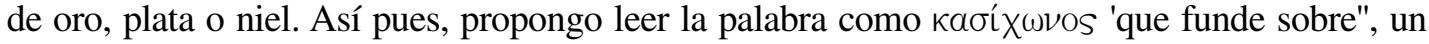

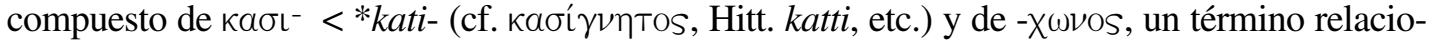
nado con $\chi \epsilon ́ \omega$, y con otros derivados que tienen que ver con la metalurgia y el trabajo de meta-

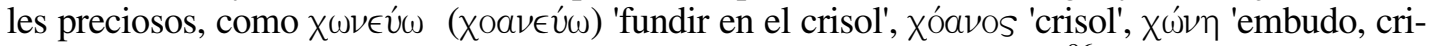
sol', y Хóvvos 'copa de cobre' (una palabra cretense según Hermonacte ${ }^{96}$ ). Pienso que no todas las formas en $\chi \omega^{-}$resultan de una contracción (cf. $\chi \omega \dot{\nu} \nu \nu v \mu$, ${ }^{\prime \prime} \chi \omega \sigma \alpha$ ) y puede tratarse de una raíz indoeuropea de las llamadas "con diptongo largo"97.

Las tablillas $\mathrm{Ra}(1)$ se refieren, pues, al tipo de dagas que conocemos bien gracias a los hallazgos arqueológicos y que tienen empuñaduras de marfil unidas por remaches y materiales incrustados en la hoja.

Frente a las tablillas de la serie Ra (1), que tienen una sola línea, en las de Ra (2) después de la primera palabra, escrita en signos más grandes, el texto se distribuye en dos líneas con una anotación diferente en cada una.

En la línea superior se anotaba un arma cuyo nombre hemos perdido en todos los $\operatorname{casos}^{98}$. En la tablilla mejor conservada (1028), pa-ka-na se omite. Parece probable que $p a-k a-n a$ se omitiera siempre porque para el escriba era bastante la presencia del logograma PUG (una daga con la punta hacia arriba). El escriba registra también e-re-pa-te de-de-me-na, i. e. É $\lambda \in \phi \dot{v} \nu \tau \in \mathrm{L}$ dativo

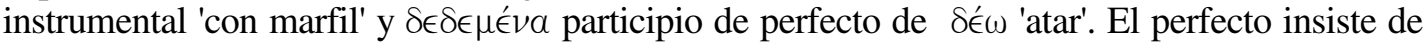
nuevo en el carácter de resultado, de obra terminada. Es probable que e-re-pa-te de-de-me-na anote la misma circunstancia que de-so-mo de la mano 126. Podría haber presentado ambas en la misma columna. Cf. É $\lambda € \phi \alpha \nu T o ́ \delta \in T O S^{99}$, que está con e-re-pa-te de-de-me-na en lamisma relación que ka-ko-de-ta (KN So 894.2) con ka-ko de-de-me-no (PY Sa 794) ${ }^{100}$. A diferencia de la mano 127, en las tablillas $\mathrm{Ra}(1)$ el escriba omite el nombre del material e-re-pa-te. La razón puede ser que para el escriba 127 el material es relevante porque en la línea inferior se anota otro material (ke-ra); en cambio, para el 126 es irrelevante, porque sabe que el material de la empuñadura de las pa-ka-na es siempre marfil (e-re-pa-te).

En la línea inferior encontramos el nombre de arma zo-wa; parece que siempre es calificada con e-pi-zo-ta. Es claro que zo-wa es el nombre del arma referida por el ideograma GUP, una daga con la punta hacia abajo. Las cantidades de este objeto son altas; en un caso (1028), la más alta, 99. Y es evidente que los objetos referidos por los logogramas GUP y PUG no son el mismo. $z o$-wa aparece también en KN Nc 4473, junto a tu-ri-jo, que parece ser un antropónimo, y en KN $\mathrm{X} 766$, junto a a-ze-to ${ }^{101}$. Prima facie, zo-wa es un nombre, mientras e-pi-zo-ta y a-ze-to son

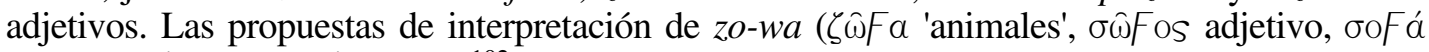

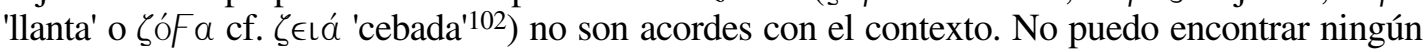
nombre griego de arma aceptable. Lo más sencillo es pensar en un nombre prehelénico perdido, pero es imposible demostrarlo.

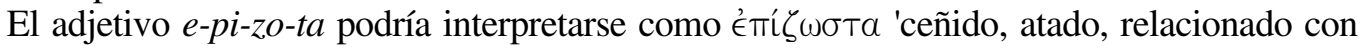
$\epsilon \in \zeta \zeta \omega ́ \nu \nu v \mu l$, en este caso las empuñaduras irían sujetas con cuerdas. Por su parte, $a$-ze-to puede

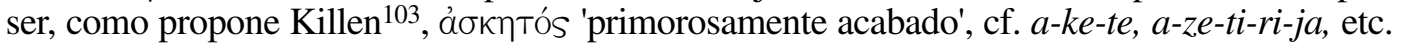

\footnotetext{
96 Hermonax in Ath.11.502b.

97 Cf. F. R. Adrados, Estudios sobre las sonantes y laringales indoeuropeas, Madrid 1973, App. A 37 $* g h e H^{w}{ }^{-}$

98 No tengo una explicación satisfactoria para la sílaba -rị que se lee en 7498.

99 Eur. Iph. Aul. 582, Aristoph. Av. 219.

100 Cf. A. Bernabé et al., "Estudios ... I", cit. p. 144 s.

101 In TH Av 100.4 zo-wa parece ser un nombre de persona.

102 Cf. DMic. s.v.

103 J. T. Killen, "The Knossos Ld(1) tablets", en E. Risch - H. Mühlestein (eds.), Colloquium Mycenaeum, Neuchâtel 1979, 167.
} 
Si e-pi-zo-ta es un neutro plural, zo-wa debe ser un neutro plural. Pero a-ze-to no puede concertar con zo-wa in KN X 766.1, donde leemos zo-wa a-ze-to e-[ (esperaríamos a-zeta). Probablemente $a$-ze-to consertaba con la palabra siguiente, quizá una parte de la $z o-w a$, por ejemplo, un dativo o instrumental plural ' $z o$-wa con x primorosamente acabados'.

El sintagma ke-ra de-de-me-na es paralelo al de la línea superior, pero ahora con el dativo o el instrumental de Ké $\rho a s$ 'cuerno', un material más barato, lo que podría explicar que las zo-wa sean más en número.

Así pues, los dos registros $\mathrm{Ra}(1)$ y $\mathrm{Ra}(2)$, tienen propósitos diferentes: el escriba 126 registra entregas de dagas ( $\phi a ́ \sigma \gamma a v a$, no de $z o-w a$ ) hechas por artesanos de dos tipos: por una

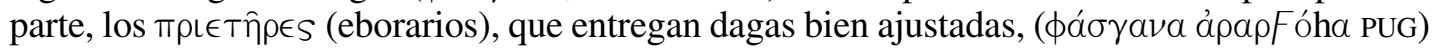

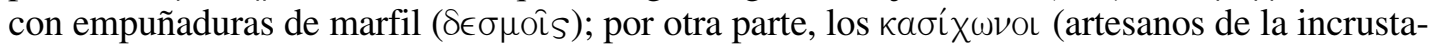
ción), que entregan dagas con otros materiales incrustados en la hoja. Es claro que el palacio había recibido previamente las hojas de los ka-ke-we y las había enviado a eborarios e incrus-

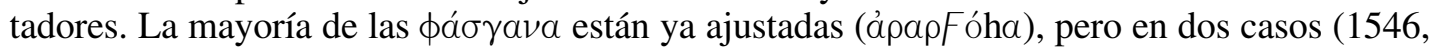
1556) parece que se ha hecho el trabajo sólo sobre la hoja, en espadas que no están ajustadas. La función del escriba 126 es, pues, asegurarse de que los artesanos entregan las dagas al palacio y comprueban si los objetos están acabados o no. Escribir de-so-mo encima de la línea le

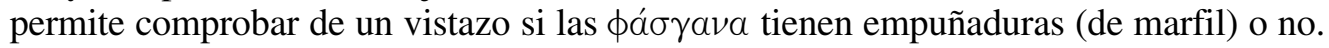

En cambio, el escriba 127 no se interesa por los trabajos específicos de los artesanos (no reseña el nombre de oficio) ni inspecciona si la obra está o no terminada (no escribe $a$-ra-ru-

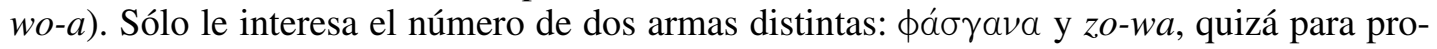
veer de armas a los combatientes de los carros, habida cuenta de qeu trabaja en el área en la que se llevan a cabo tareas con los carros.

\subsection{Otros nombres de la espada en atestiguación indirecta}

Indirectamente se atestiguan otros dos nombres de la espada, *ảh y ảhop. El primero podría presumirse a partir del antropónimo $a-i-q e-u(a-i-q e-w o, a-i-q e-w e)$ si aceptamos su

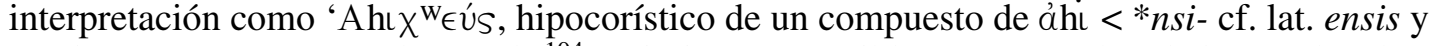
* gwhontas 'que mata con la espada'104. Asimismo es posible ver un nombre de la espada a[hor

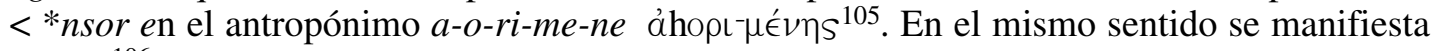
Hajnal $^{106}$, quien interpreta el antropónimo a-e-ri-qo-ta como aherik ${ }^{w} h o n t a \bar{s}$, cuyo primer elemento sería un locativo con grado e de esta misma palabra, esto es, *aseri-107.

\subsection{0. ¿Hachas?}

Más problemático es el caso de la existencia del hacha como arma de guerra en micénico. Incluso en el caso de que pudiéramos leer pe-re-ke-u como $\pi \in \lambda \in \kappa \in U ́ s$ en PY Cn 1287.5 (lo que es más que dudoso, habida cuenta de que el contexto del mismo nombre en MY Oe 130 aconseja más bien $\pi \lambda \in \kappa \in U$ S 'tejedor') ${ }^{108}$ y admitiéramos que el difícil compuesto pe-re$k u$-wa-na-ka contiene el nombre del hacha, lo que es incluso más dudoso ${ }^{109}$, ello no implicaría necesariamente que el hacha fuera usada como arma de guerra ${ }^{110}$.

104 Cf. M. Lejeune, MPhM III, 251s. Lo duda Ferluga, "La terminologia...", cit., p. 382s. Cf. otras interpr. en DMic. s. v.

105 Cf. DMic. s.v. Para la formación cf. Sobre etimología cf. p. 299, n. 60.

106 Cf. Hajnal, "Der Mykenische...”, cit.

107 Sobre etimología cf. p. 299, n. 60.

108 Cf. DMic. s. v.

109 Cf. DMic. s. $v$.

110 Cf. Ferluga, "La terminologia...”, cit., p. 383s. 


\section{CONCLUSIONES}

En el cuadro II puede visualizarse la "historia resumida" de los diversos términos estudiados. Las palabras aparecen en el caso y número en que están documentadas, salvo las que están entre corchetes y sin indicación de la forma micénica, lo que indica que su atestiguación es indirecta; falta la transcripción griega cuando no podemos proponerla; ide $=$ origen indoeuropeo; comp. gr. $=$ compuesto griego sobre temas indoeuropeos; prehel. $=($ probable $)$ origen prehelénico, prést. $=($ probable $)$ préstamo; $?=$ origen desconocido. La cruz señala la situación del término como conservado, alterado o perdido.

\begin{tabular}{|c|c|c|c|c|}
\hline \multicolumn{5}{|c|}{ CUADRO II: GRADO DE CONSERVACIÓN DEL VOCABULARIO MICÉNICO: } \\
\hline término micénico & origen & conservado & alterado & perdido \\
\hline$[\theta \in u ́ x \in h \alpha]$ & ide. & + & & \\
\hline$\left[\mathrm{E}^{\prime} \nu T \in \mathrm{h} \alpha\right]$ & ide. & + & & \\
\hline$[0 ̈ \pi \lambda \alpha]$ & ide. & + & & \\
\hline$[E ̈ v a \rho a]$ & ide. & + & & \\
\hline to-ra-ka $\theta \omega \hat{\omega} a \xi$ & ? & + & & \\
\hline ko-ru-to кópvӨos & prehel. & + & & \\
\hline o-pa-wo-ta о̉тáFорта & comp. gr. & & & + \\
\hline pa-ra-wa-jo $\pi \alpha \rho \bar{\alpha} F \mathrm{~h}_{\alpha i}{ }^{\omega} \omega$ & comp. gr. & & + & \\
\hline wi-so-wo-pa-tọ Fıбота́Fорто & comp. gr. & & + & \\
\hline 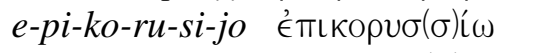 & comp. gr. & & + & \\
\hline 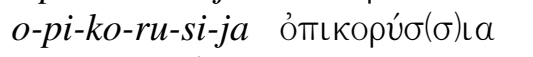 & comp. gr. & & + & \\
\hline$q e-r o_{2} \sigma \kappa^{\mathrm{w}} \in \dot{\lambda} \lambda \omega$ & ide. & & + & \\
\hline e-po-mi-jo $\epsilon^{\prime} \pi \omega \mu \mathrm{h}_{\mathfrak{L}} \omega$ & comp. gr. & + & & \\
\hline$[\tau o ́ \xi \alpha]$ & prést. & + & & \\
\hline$a_{3}-k a-s a-m a$ aik $\sigma \mu a ́ v s$ & ide. & + & & \\
\hline$e$-Jke-a, e-ke-si É $^{\prime} \gamma \in \mathrm{h} \alpha,-\sigma \mathrm{\iota}$ & prést. & + & & \\
\hline pa-ta-ja па入таîa & ide. & & + & \\
\hline [äkovTes] & ide. & + & & \\
\hline$\left[\gamma^{\mathrm{w}} \in \dot{\lambda} \lambda \in \mu \nu \alpha\right]$ & ide. & + & & \\
\hline [íoú] & ide. & + & & \\
\hline$w a-o$ & prehel. & & & + \\
\hline 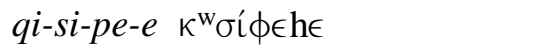 & $?$ & + & & \\
\hline 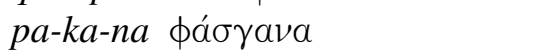 & $?$ & + & & \\
\hline$z o-w a$ & $?$ & & & + \\
\hline 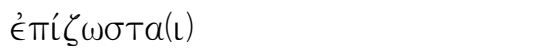 & comp. gr. & & & + \\
\hline ảoknтós & $?$ & + & & \\
\hline àpapFóha & ide. & + & & \\
\hline$\delta \in \delta \in \mu \epsilon ́ v a$ & ide. & + & & \\
\hline$\delta \in \sigma \mu o \hat{s}$ & ide. & & + & \\
\hline 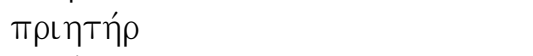 & $?$ & & & + \\
\hline 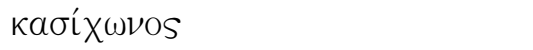 & comp. gr. & & & + \\
\hline [ähl] & ide. & & & + \\
\hline [ähop] & ide. & + & & \\
\hline
\end{tabular}


A la luz del cuadro II advertimos que con respecto al origen y fortuna posterior del vocabulario micénico de las armas pueden diferenciarse claramente tres tipos de términos:

a) Un primer grupo lo forman términos muy genéricos, para los que se usan palabras de léxico corriente, derivadas de raíces indoeuropeas, no especializadas, como demuestra su

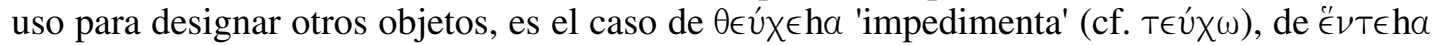

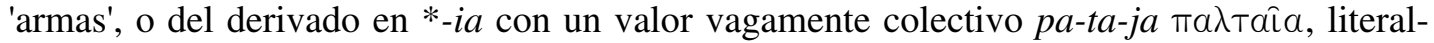
mente, 'cosas que se blanden', $\gamma^{\mathrm{w}} \in \dot{\lambda} \epsilon \mu \nu \alpha$ 'cosas que se lanzan' o bien öm $\lambda \alpha$ 'cosas que se manejan' o, si realmente existe en micénico, évapa 'lo que se obtiene como botín'. Cf. tam-

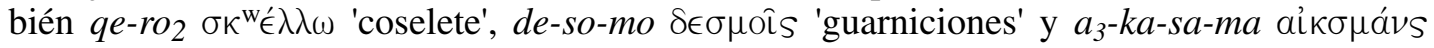
'puntas'. Son también del léxico corriente los participios de perfecto $a$-ra-ru-wo-a ảpapF óha, 'ajustadas' y de-de-me-na $\delta \in \delta \in \mu \epsilon ́ v a$ 'guarnecidas'. De ellos continúan usándose con el

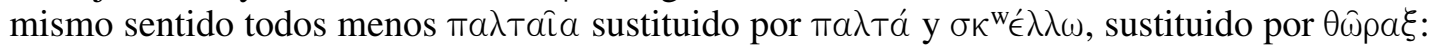
la designación de la 'armadura' se especializa en designar la coraza y desaparece el antiguo término.

Como suele suceder, cuanto más genérico es un nombre micénico, más fácil es que traspase la barrera del segundo al primer milenio.

Es curioso reseñar que varios términos antiguos, de raigambre indoeuropea no aparecen en micénico más que en forma residual, formando parte de nombres propios o de topónimos: es el caso de los nombres genéricos öt $\lambda \alpha$ y évapa (aunque este último es más lógico que no aparezca en los archivos de palacio), dos designaciones de la espada, *ảhı- y ähop y los nombres de armas arrojadizas ăk $\omega \nu$, iós y $\gamma^{\mathrm{w}} \in \hat{\epsilon} \lambda \in \mu \nu \mathrm{O \nu}$.

Después hallaremos åop en Homero y como homerismo en algunos poetas posteriores.

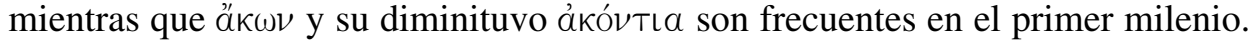

b) Un segundo grupo lo forman los términos básicos para las armas concretas, que son, en su mayoría, de origen no indoeuropeo, y casi siempre desconocido: to-ra(-ke) $\theta \omega \dot{p} a \xi, \theta \omega^{-}$

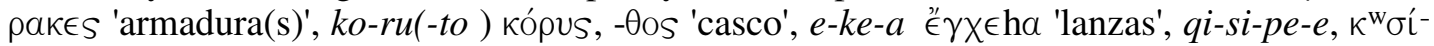

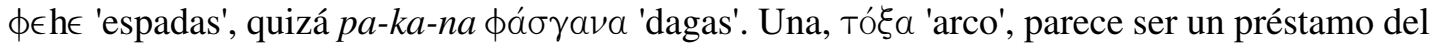
iranio. En dos casos, wa-o 'doble hacha' y zo-wa un tipo de 'dagas' desconocemos incluso la forma concreta que tendría la palabra. Salvo estos dos últimos casos (que obviamente se han perdido en el griego del primer milenio), los demás continuaron en uso, al menos en Homero y varios de ellos, mucho después. Sea cual fuere el origen de estos términos, es claro que el nombre acompañaba a un arma nueva, resultado de una determinada innovación tecnológica, convirtiéndose luego durante mucho tiempo en designación genérica.

c) El tercer grupo lo constituyen términos para designar, bien partes de las armas, bien calificativos, bien oficios, que muestran los procedimientos de formación del griego. Se trata en general de neologismos, en el primer caso, casi todos compuestos, que aluden a la posición que ocupa la pieza que designan con respecto a la parte del cuerpo que cubren o con respecto a otra parte de la propia armadura:

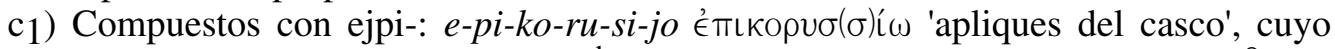

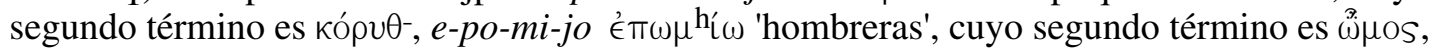

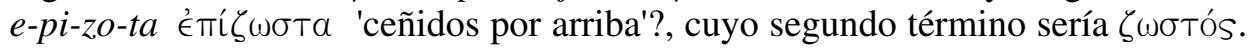

c2) Compuestos con ojpiv: o-pa-wo-ta ómáF орта 'apliques, placas', cuyo segundo tér-

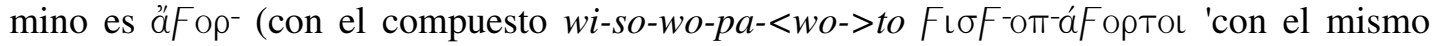

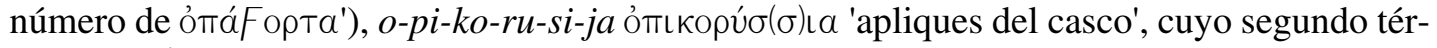
mino es кópu $\theta^{-}$. es aủ $\sigma^{-}$.

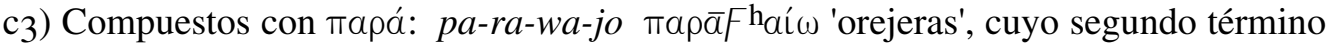

c4) Otros procedimientos de formación, propios del griego, aparecen en nombres de

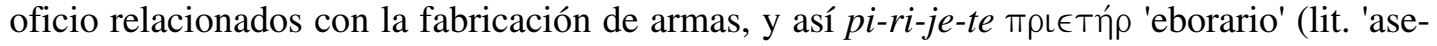
rrador') es un nombre de agente derivado sobre el verbo $\pi \rho i ́ \omega$ (si bien su etimología es des- 


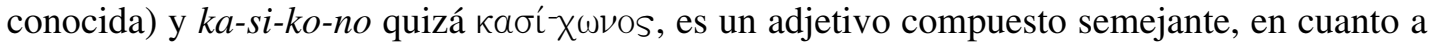

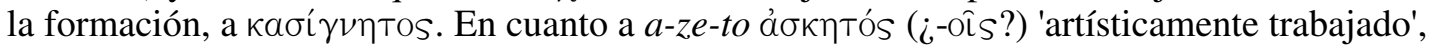

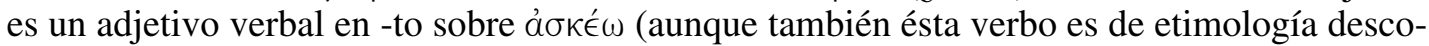
nocida); cf. los nombres de agente masculino y femenino, documentados en micénico, $a$-ke-

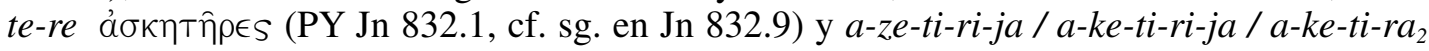

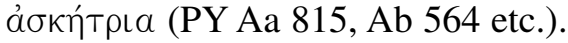

Los neologismos de este tercer grupo han desaparecido en su totalidad. Sólo se con-

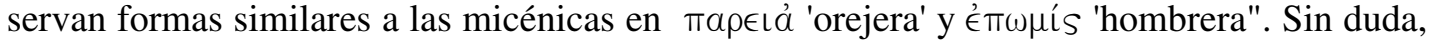
modificaciones en la estructura de las armas y/o en el proceso de fabricación motivaron el olvido y posterior pérdida de la mayoría de este rico y preciso vocabulario relacionado con las armas.

Termina así esta panorámica sobre la terminología micénica de las armas, que creo que puede suministrar unas bases sobre las que comparar los hallazgos de la arqueología y la información brindada por la iconografía.

Recibido: 06/07/2007

Aceptado: 20/07/2007 